\title{
Stochastic Modelling of Insulin Sensitivity and Adaptive Glycemic Control for Critical Care
}

\author{
Jessica Lin ${ }^{1}$, Dominic Lee ${ }^{2}$, J. Geoffrey Chase ${ }^{3}$, Geoffrey M. Shaw ${ }^{4}$, \\ Aaron Le Compte ${ }^{1}$, Thomas Lotz ${ }^{1}$, Jason Wong ${ }^{1}$, Timothy Lonergan ${ }^{1}$, \\ Christopher E. Hann ${ }^{5}$
}

${ }^{1}$ Research Assistant, Dept of Mechanical Engineering, Centre for Bio-Engineering, University of Canterbury, Private Bag 4800, Christchurch, NZ

${ }^{2}$ Sr. Lecturer, Dept of Mathematics and Statistics, University of Canterbury, Private Bag 4800, Christchurch, NZ

${ }^{3}$ Professor, Dept of Mechanical Engineering, Centre for Bio-Engineering, University of Canterbury, Private Bag 4800, Christchurch, NZ, Email: geoff.chase@canterbury.ac.nz

${ }^{4}$ Consultant and Sr. Lecturer, University of Otago, Christchurch School of Medicine and Health Sciences, Christchurch, NZ, and Canterbury District Health Board, Dept of Intensive Care Medicine, Christchurch Hospital

${ }^{5}$ Post-Doctoral Fellow, Dept of Mechanical Engineering, Centre for Bio-Engineering, University of Canterbury, Private Bag 4800, Christchurch, NZ 


\begin{abstract}
Targeted, tight model-based glycemic control in critical care patients that can reduce mortality $18-45 \%$ is enabled by prediction of insulin sensitivity, $S_{I}$. However, this parameter can vary significantly over a given hour in the critically ill as their condition evolves. A stochastic model of $S_{I}$ variability is constructed using data from 165 critical care patients. Given $S_{I}$ for an hour, the stochastic model returns the probability density function of $S_{I}$ for the next hour. Consequently, the glycemic distribution following a known intervention can be derived, enabling pre-determined likelihoods of the result and more accurate control.
\end{abstract}

Cross validation of the $S_{I}$ variability model shows that $86.6 \%$ of the blood glucose measurements are within the 0.90 probability interval, and $54.0 \%$ are within the interquartile interval. "Virtual Patients" with $S_{I}$ behaving to the overall $S_{I}$ variability model achieved similar predictive performance in simulated trials (86.8\% and $45.7 \%)$. Finally, adaptive control method incorporating $S_{I}$ variability is shown to produce improved glycemic control in simulated trials compared to current clinical results. The validated stochastic model and methods provide a platform for developing advanced glycemic control methods addressing critical care variability.

Keywords: stochastic modelling, insulin sensitivity, blood glucose, intensive care, adaptive control, probability intervals, control protocol simulations 


\section{Introduction}

Critically ill patients often experience stress-induced hyperglycemia and high levels of insulin resistance [1-4]. The increased counter-regulatory hormone and cytokine response stimulates endogenous glucose production and increases effective insulin resistance. Absolute and relative insulin deficiency and steroid-based therapies are further causes. The metabolic response to stress is characterised by highly variable changes in insulin sensitivity and glucose metabolism. The overall result is hyperglycemia, the pathogenesis of which is becoming well described $[1,5]$. Tight glucose control has been shown to reduce Intensive Care Unit (ICU) patient mortality up to $45 \%$.

Previous clinical model-based glycemic control studies include [6-12]. Chase et al. [10] and Plank et al. [8] developed highly adaptive, clinically verified, targeted control algorithms. Following [10], the specialized relative insulin and nutrition tables (SPRINT) protocol $[7,11]$ was developed to simplify critical care implementation. Over 165 patients and 23,000 patient hours, it achieved an average blood glucose level of $5.8 \pm 1.2 \mathrm{mmol} / \mathrm{L}$ with $61 \%$ of the time in the $4-6.1 \mathrm{mmol} / \mathrm{L}$ band [4]. This is the only approach adaptive to insulin sensitivity variation, as reflected in the hour to hour insulin-nutrition response to changing blood glucose levels.

A common risk in intensive insulin therapy is hypoglycemia. Many current ad-hoc intensive insulin therapy protocols have reported hypoglycemic episodes for up to $25 \%$ of patients $[3,13]$. Therefore, understanding and modelling the variability in insulin sensitivity can assist clinical control intervention and minimise associated risk. 
Currently, no intensive insulin therapy protocol offers the glycemic response likelihood to an intervention. Clinicians are thus partly blind in controlling this highly dynamic system. Therefore, the goal of this study is to produce blood glucose probability distributions for control intervention decisions based on stochastic models of clinically observed parameter variations. The result will allow more accurately targeted control, with user specified likelihood on the glycemic response.

\section{Glucose-Insulin System Model and Parameter Identification}

A clinically verified patient-specific glucose-insulin metabolic model $[10,12,14,15]$ is used to account for time-varying insulin sensitivity in critical care.

\subsection{Glucose-insulin system model}

The Glucose-insulin system model is presented in Equations (1)-(5).

$$
\begin{aligned}
& \dot{G}=-p_{G} G-S_{I}\left(G+G_{E}\right) \frac{Q}{1+\alpha_{G} Q}+P(t) \\
& \dot{Q}=-k Q+k I \\
& \dot{I}=-n \frac{I}{1+\alpha_{I} I}+\frac{u_{e x}(t)}{V_{I}} \\
& P\left(t_{i}<t<t_{i+1}\right)=\bar{P}_{i+1}+\left(P\left(t_{i}\right)-\bar{P}_{i+1}\right) e^{-k_{p d}\left(t-t_{i}\right)} \text { where } \bar{P}_{i+1}<P\left(t_{i}\right) \\
& P\left(t_{i}<t<t_{i+1}\right)=\bar{P}_{i+1}+\left(P\left(t_{i}\right)-\bar{P}_{i+1}\right) e^{-k_{p r}\left(t-t_{i}\right)} \text { where } \bar{P}_{i+1}>P\left(t_{i}\right)
\end{aligned}
$$

where $G[\mathrm{mmol} / \mathrm{L}]$ denotes glucose above an equilibrium level $G_{E}[\mathrm{mmol} / \mathrm{L}]$, and $I$ $[m U / L]$ denotes the plasma insulin from an exogenous insulin input. Insulin utilization over time is captured by $Q[\mathrm{mU} / L]$, where $k[1 / \mathrm{min}]$ is the effective insulin half-life 
parameter. Patient endogenous glucose removal and insulin sensitivity are $p_{G}[1 / \mathrm{min}]$ and $S_{I}[L / m U / m i n]$ respectively. The parameter $V_{I}[L]$ is the insulin distribution volume and $n[1 / \mathrm{min}]$ is the first order decay rate for plasma insulin. External nutrition and insulin inputs are $P(t)[\mathrm{mmol} / \mathrm{L} / \mathrm{min}]$ and $u_{e x}(t)[\mathrm{mU} / \mathrm{min}]$, respectively. Michaelis-Menten parameters $\alpha_{I}[L / m U]$ and $\alpha_{G}[L / m U]$ define plasma insulin disappearance saturation and insulin-stimulated glucose removal saturation, respectively. The exogenous glucose appearance and decay rate used to define $P(t)$ are $k_{p r}$ and $k_{p d}$. Specific details on physiological model dynamics are evaluated in [10, $12,16]$.

In Equation (1), the saturation mechanism on insulin effect creates a unique index of insulin sensitivity, $S_{I}$, compared to other model-based measures. The result is an $S_{I}$ index that more closely approximates the effective net tissue sensitivity to insulin, and its variation to the evolution of patient condition and drug therapy. It has also been shown to drive patient response to intervention and thus control efficacy in prior studies $[10,12]$. This model measure is also highly correlated to clamp-derived ISI over 146 patients [17-19]. Hence, understanding variation in $S_{I}$ should enable more effective control.

\subsection{Integral-based parameter identification}

Identifying insulin sensitivity has been extensively studied [1, 20]. Using constant population values for $p_{G}, \alpha_{G}, \alpha_{I}, n, k$ and $V_{I}$ [15] limits the model unknowns in this case to $S_{I}$. This study utilizes an integration-based parameter identification method first presented in Hann et al. [15]. Constraints are placed on $S_{I}$ to ensure it is within a physiologically valid range. 


\section{Stochastic Modelling and Validation}

The control algorithms of $[10,12]$ calculate the interventions necessary for targeted glycemic regulation by assuming that the currently identified $S_{I}$ values are constant between the control intervention and the one-hour time interval to achieve a preselected target at the next measurement. This assumption is made due to lack of better knowledge. However, $S_{I}$ can evolve significantly [15], and sudden variations may occur due to the onset of acute conditions [12].

The ultimate goal is to produce blood glucose probability bands for a given intervention based on clinically observed parameter variations using an ICU population based stochastic model. Such bands would allow glycemic target selection with guaranteed levels of certainty that a result will meet or exceed a given glycemic level. Such a model would also identify outlying measurements to a pre-defined probability level.

An initial such model was developed using data from 18 critical care patients in the Department of Intensive Care Medicine (ICU), Christchurch Hospital [21]. It was clinically verified with data from 8 independent critical care glycemic control trials. The model successfully captured the identified the $S_{I}$ variation trend, accounting for $84 \%$ of measurements within the 0.90 probability interval, and $45 \%$ within the interquartile interval. However, it was limited in its formulation from a population limited in size $(n=18)$, the resulting level and variety of critical illness, and the glucose data density ( $1-4$ hourly). 
Between July 2005 and June 2006, 165 patients were put on the SPRINT protocol in the Christchurch Hospital ICU [11]. A new stochastic model was created using data from these 165 controlled patients, totalling over 23,000 hours, to better represent stochastic $S_{I}$ variation in critical care. By comparison, the initial model was only built on close to 1,300 hours of data over 18 patients, none of whom were on intensive glycemic control. The SPRINT blood glucose data were primarily hourly data, whereas the previous model was built from $3-4$ hourly data. Therefore, SPRINT data provides a better view of the highly dynamic critically ill condition that is more suitable for intensive glycemic control.

\subsection{Stochastic $S_{I}$ model for critical care}

Patient specific insulin sensitivity parameter profiles, $S_{I}$, are fitted hourly to the 165 SPRINT patients. Table 1 shows the patient data. Glycemic control had greatest effect for those with greater than 3-day stay $[3,13]$ and they make up $73 \%$ of this cohort and $96 \%$ of the patient hours.

Fitted $S_{I}$ is constrained between physiological limits of $1 \times 10^{-5}$ and $1 \times 10^{-3}(\mathrm{mU} / \mathrm{L} / \mathrm{min})$ $[22,23]$. A 2-dimensional kernel density estimation method is used to construct the stochastic $S_{I}$ model that describes the hourly transition of parameter values. The method has the advantage of producing a smooth, continuous function across the parameter range [24]. The overall result is a bivariate probability density function for the potential parameter values. Figure 1 shows the distribution of fitted hour to hour $S_{I}$ and the 2-dimensional kernel joint probability density. 
The 2-dimensional kernel density method is chosen for creating the stochastic model because the distribution of $S_{I}$ at hour $n+1\left(S_{I n+1}\right)$ varies with $S_{I}$ at hour $n\left(S_{I n}\right)$, and cannot be simply described with a standard statistical distribution. Thus, the variations in $S_{I}$ can be treated as a Markov process. A Markov process has the property that the conditional probability density function of future states of the process, given the present state, depends only upon the current state. Therefore, using the Markov property of the stochastic behaviour of $S_{I}$, the conditional probability density of $S_{I n+1}$ taking on a value $y$ can be calculated by knowing $S_{I n}=x$ :

$$
p\left(S_{I n+1}=y \mid S_{I n}=x\right)=\frac{p\left(S_{I n}=x, S_{I n+1}=y\right)}{p\left(S_{I n}=x\right)}
$$

Considering the fitted $S_{I}$ in a 2-D space, as shown in Figure 1, the joint probability density function across the $x-y\left(S_{I n}-S_{I n+1}\right)$ plane is defined by the fitted values shown by the dots whose coordinates are $x_{i}$ and $y_{i}$,

$$
\begin{aligned}
& p(x, y)=\frac{1}{n} \sum_{i=1}^{n} \frac{\phi\left(x ; x_{i}, \sigma_{x_{i}}{ }^{2}\right)}{p_{x_{i}}} \frac{\phi\left(y ; y_{i}, \sigma_{y_{i}}{ }^{2}\right)}{p_{y_{i}}} \\
& p_{x_{i}}=\int_{0}^{\infty} \phi\left(x ; x_{i}, \sigma_{x_{i}}{ }^{2}\right) \\
& p_{y_{i}}=\int_{0}^{\infty} \phi\left(y ; y_{i}, \sigma_{y_{i}}{ }^{2}\right)
\end{aligned}
$$

Effectively, the 2-D joint probability density function is the normalised summation of normal probability density functions $\phi\left(x ; x_{i}, \sigma_{x_{i}}{ }^{2}\right)$ centred at individual data points. 
In Equations (7)-(9), the variance, $\sigma$, at each data point is a function of the local data density in a centred and orthonormalised space of $x$ and $y$. Putting Equations (8) and (9) into Equation (7) normalises each $\phi\left(x ; x_{i}, \sigma_{x_{i}}{ }^{2}\right)$ and $\phi\left(y ; y_{i}, \sigma_{y_{i}}{ }^{2}\right)$ in the positive domain, effectively putting boundaries along $x=0$ and $y=0$, and enforcing physiological validity in $S_{I}$ values.

In Equation (6), the right hand side denominator can be calculated by integrating Equation (7) with respect to $y$. Hence, Equation (7) can be calculated:

$$
\begin{aligned}
& p\left(S_{I n+1}=y \mid S_{I n}=x\right)=\sum_{i=1}^{n} \omega_{i}(x) \frac{\phi\left(y ; y_{i}, \sigma_{y_{i}}{ }^{2}\right)}{p_{y_{i}}} \\
& \omega_{i}(x)=\frac{\phi\left(x ; x_{i}, \sigma_{x_{i}}{ }^{2}\right) / p_{x_{i}}}{\sum_{j=1}^{n} \phi\left(x ; x_{j}, \sigma_{x_{j}}{ }^{2}\right) / p_{x_{j}}}
\end{aligned}
$$

Thus, knowing $S_{I n}=x$ at hour $n$, the probability of $S_{I n+1}=y$ at hour $n+1$ can be calculated using Equations (10) and (11). The probability intervals in Figure 1 are also calculated from integrating Equation (10). Plotting Equation (10) across the $x-y\left(S_{I n}\right.$ $\left.S_{I n+1}\right)$ plane, the resulting 3-D stochastic model is shown in Figure 2. This essentially creates an approximation to the parameter variation behaviour according to how the existing data behaves. Where there is higher density of data, more certainty can be drawn on the "true" behavioural pattern. With this stochastic model, given a current identified $S_{I}$ value, the probability density can be calculated across the possible range of $S_{I}$ values for the coming hour. The probability is conditional because the probability density for a coming hour is dependent on the identified current hourly $S_{I}$. 
Across the fitted range of $S_{I}$, the conditional probability density function is largely uni-modal and symmetric. However, as shown in the cascade plot of conditional $S_{I n+1}$ probability density functions across the $S_{I n}$ model range in Figure 3, there are some numerical artefacts near the boundaries of the fitted range. Probability density functions for $S_{I n}$ peak at boundary values because of the fitting constraints. For $S_{I n}=$ $\left[1 \times 10^{-5}, 1 \times 10^{-4}\right]$ and $\left[0.75 \times 10^{-3}, 1 \times 10^{-3}\right]$ at the edges of Figure 3 , the percentile values away from the median do not necessarily decrease in probability. These ranges span $34 \%$ of the fitting range $\left[1 \times 10^{-5}, 1 \times 10^{-3}\right]$, in which $20 \%$ of the SPRINT data falls $\left(18 \%\right.$ between $S_{I n}=\left[1 \times 10^{-5}, 1 \times 10^{-4}\right]$ and $2 \%$ between $\left[0.75 \times 10^{-3}, 1 \times 10^{-3}\right]$ in Figure 1). Note that Figures 2 and 3 show the conditional probability density functions which are scaled to have the area under each function summing to 1 . Thus, the asymmetry becomes very pronounced in Figures 2 and 3. The probability of $S_{I}$ taking on these asymmetric conditional probability density functions is in fact very low in the overall joint probability density function shown in Figure 1, where the probability density sums to 1 over the entire fitting surface.

The asymmetric $S_{I}$ probability density functions could perhaps be due to other not explicitly modelled physiology, such as variations in endogenous glucose production. More specifically, "bumpy" areas of $S_{I}$ probability density function in Figure 3 may be a sign of $S_{I}$ absorbing physiology unmodelled or undermodelled in Equations (1)(5). However, this unmodelled physiology represents uncommon sudden, or extreme, dynamics, evident by the low overall joint probability density in these areas in Figure 1. Practically, to include all such variations in the glucose-insulin model would make it overly complicated and it would thus lose clinical feasibility. Such additions would 
also require added assumptions for endogenous insulin or glucose production that are not clinically measured in real-time.

Assumptions for these responses could also compromise control accuracy, as both endogenous insulin and glucose productions are known to vary significantly between patients [25-29] and over time. In addition, endogenous glucose production is suppressed with significant insulin administration in both normal and stressed states [30], which is the case for critically ill patients under insulin therapy for glycemic management. Similarly, the endogenous insulin production is effectively removed or significantly reduced in the presence of significant exogenous insulin [29, 31-33], not to mention its inhibition in stress hyperglycemia [e.g. 34]. It is therefore, in the interest of this model-based clinical control applications, to have these effects mitigated into $S_{I}$. In $[10,12,15]$, the model of Equations (1)-(5) and the impact of mitigated effects are discussed in detail. The result is a much more clinically feasible control model, where $S_{I}$ accounts for critically ill population's overall sensitivity to insulin and/or its utilisation.

In Figure 1, the lower bound for $S_{I}$ has greater influence on the overall stochastic model than the upper bound. However, no patients have fitted $S_{I}$ staying at the lower bound for a prolonged period, again suggesting that these occasions are more sudden, short term condition, such as suppressed insulin sensitivity or utilisation due to drug therapies, rather than long term, or gradual changes, such as increased glucose production. During numerical fitting, if fitted $S_{I}$ stays at the lower bound for a prolonged period of 5 hours, the fitting method recognises a gradual evolution, and adjusts $G_{E}$ to address factors such as endogenous glucose production. During the total 
of 23,324 control hours, only 39 such occurrences were observed, which is $0.17 \%$ of the time.

The short term changes, although causes fitted $S_{I}$ to hit the lower bound, if unconstraint, will often result in negative $S_{I}$ because of significant drug effects or acute medical conditions such as atrial fibrillation [12]. In addition, increased endogenous production with inhibited glucose production can result in an effectively zero or negative modelled $S_{I}$. These cases are too wide ranged to be accounted for in the physiological model. Note that an evolving $G_{E}$ that is too high leads to a reduction in modelled endogenous glucose production, and a higher $S_{I}$ may also result in the model due to a lower $-p_{G} G$ term. Mitigating the impact of these events into fitted $S_{I}$ simplifies the model to be clinically control feasible.

In addition, incorrect modelling of these conditions which are difficult to account for can severely compromise patient safety. More specifically, underestimating $S_{I}$ can lead to excess insulin being given. With the aim of applying the physiological and stochastic models in clinical control, the lower bound of $S_{I}$ has an important role. Finally, the fitted $S_{I}$, with higher data concentration around the lower bound, realistically reflects the highly variable dynamics in the critical care environment, where drug therapies and acute medical conditions that result in highly resistant patients are common.

\subsection{Clinical implementation and use}

Having constructed the $S_{I}$ stochastic model, the surface of Figure 2 can be used in a look-up table. Given a clinically identified $S_{I}[10,12]$, the probability density, and 
hence the probability intervals, can be obtained, as demonstrated in Figure 4. The solid line is the kernel density estimate surface sliced along $S_{I n}=0.6 \times 10^{-3}$. This line represents the probability density for potential $S_{I n+1}$, one hour after having identified the current hour $S_{I n}=0.6 \times 10^{-3}$. From this density function, probability intervals are also obtained, giving a median probable $S_{I}$ value in an hour of $0.58 \times 10^{-3}$, interquartile range $\left[0.49 \times 10^{-3}, 0.66 \times 10^{-3}\right]$, and the 0.90 probability interval $\left[0.33 \times 10^{-3}\right.$, $\left.0.79 \times 10^{-3}\right]$.

The probability intervals used here for clinical decision making, as shown in Figure 4, are equal-tailed. "Equal-tailed" means that the 0.90 probability interval is between the $5^{\text {th }}$ percentile and the $95^{\text {th }}$ percentile in the probability density function. Equaltailed probability intervals are based on the assumption and observation that the probability density function is (largely) uni-modal and symmetric. Thus, the values outside the interval are assumed to have lower probability of occurring than the values within the interval and represent "tails" of that distribution.

\subsubsection{Probability interval calculation}

Using the equal-tailed probability interval boundary values for $S_{I}$ in Equations (1)-(5), the corresponding probability intervals in blood glucose levels for a given intervention from current state can be calculated. This statistical approach holds for any strictly monotone physiological systems [35]. More specifically, in this case, a higher $S_{I}$ value always produces lower blood glucose than a lower $S_{I}$ value for a given input and state over the time $S_{I}$ is defined. 
In the cascade plot of the conditional $S_{I n+l}$ probability density functions across the $S_{I n}$ range in Figure 3, it is evident that the assumption that the conditional probability density function is largely uni-modal and symmetric is true for the bulk of the fitted $S_{I}$ range, but does not hold near the boundaries. Therefore, in these regions, equal-tailed probability intervals may not give an exact representation of the probability intervals. More simply, the $5^{\text {th }}$ and the $95^{\text {th }}$ percentile in the resulting blood glucose level probability distribution may not contain the exact $90 \%$ most probable blood glucose levels. In particular, due to the asymmetric boundary density functions in $S_{I}$, blood glucose levels outside this range may have a higher probability density than parts within the 0.90 probability interval.

To exactly obtain the probability density in blood glucose resulting from a known probability density function in $S_{I}$, Monte Carlo simulation is the only method. In particular, while the percentile values in $S_{I}$ correspond to percentile values in blood glucose levels, the "rank" of probability does not [35].

This situation is illustrated in Figure 5, where the left hand side and right hand side demonstrate the difference between the 0.90 equal-tailed blood glucose probability intervals and the exact probability intervals generated from Monte Carlo simulations. Panels A and B display the same probability density function (pdf) (right axis) and the cumulative distribution functions (cdf) (left axis) in $S_{I n+l}$, when $S_{I n}=8 \times 10^{-4}$ is known. Panels $\mathrm{C}$ and D show the resulting pdf (right axis) and cdf (left axis) in blood glucose levels at hour $n+1\left(B G_{n+1}[\mathrm{mmol} / \mathrm{L}]\right)$ through Monte Carlo Simulation of the model in Equations (1)-(5). The pdf and cdf shown in panels C and D are identical and are the exact solution for the $B G_{n+1}$ probability density function given $S_{I n}=8 \times 10^{-4}$. Note that 
in panels $\mathrm{A}$ and $\mathrm{B}$, the $\mathrm{x}$-axes are decreasing from left to right, producing a similar shaped probability density function in blood glucose levels in panels C and D with blood glucose levels increasing as $S_{I n+l}$ decreases.

The left hand side of Figure 5, panels $\mathrm{A}$ and $\mathrm{C}$ illustrate how equal-tailed probability intervals in $S_{I n+l}$ are translated into the equal-tailed probability intervals in $B G_{n+l}$. Let $f$ be the transformation function between $S_{I n^{+1}}$ to $B G_{n+1}$, which is the physiological model shown in Equations (1)-(5), then

$$
B G_{100 \text {-percentile }}=f\left(S_{\text {Ipercentile }}\right)
$$

Thus, the percentile values of $S_{I n+I}$ in panel A corresponds to the reversed (100percentile) values in $B G_{n+1}$ in panel C. Or more simply, solving Equations (1)-(5) using the $5^{\text {th }}$ percentile value in $S_{I n}$ produces the $95^{\text {th }}$ percentile blood glucose levels, $B G_{95}=f\left(S_{I 5}\right)$ given that $f$ is strictly monotone [35]. Therefore, the $S_{I n+l}$ probability interval between the $5^{\text {th }}$ and the $95^{\text {th }}$ percentile $\left(S_{I n+1}=\left[0.33 \times 10^{-3}, 0.98 \times 10^{-3}\right]\right.$ between $\triangleright$ and $\triangleleft$ in panel A) consequently gives the $B G_{n+1}$ probability interval between the $5^{\text {th }}$ and the $95^{\text {th }}$ percentile $\left(B G_{n+1}=[1.9,5.4]\right.$ between $\triangleright$ and $\triangleleft$ in panel C). More simply, as shown in the left hand side of Figure 5,

- $\quad$ Equal-tailed 0.90 probability interval in $S_{I n+l}=\left[S_{I 5}, S_{I 95}\right]$

- $\quad$ Equal-tailed 0.90 probability interval in $B G_{n+1}=\left[f\left(S_{I 95}\right), f\left(S_{I 5}\right)\right]$

However, as illustrated in panel $\mathrm{C}$, the $B G_{n+1}$ probability interval between the $5^{\text {th }}$ and the $95^{\text {th }}$ percentile $(\triangleright$ and $\triangleleft$ ) includes values that have lower probability density than 
some $B G_{n+l}$ values outside this interval. More specifically, $\triangleright$ has lower probability density than the region to its left. This higher probability density region outside the $5-95 \%$ range is very narrow and at the very extreme end.

On the right hand side of Figure 5, the $90 \%$ most probable $S_{I n+I}$ values are identified by the shaded areas in panel B. These values are discontinuous, and have higher probability of occurring than values outside the shaded areas. However, taking the boundary values for the $90 \%$ most probable $S_{I n+l}$ values then putting them into Equations (1)-(5) does not provide the $90 \%$ most probable $B G_{n+l}$ intervals. More simply, as shown in the right hand side of Figure 5,

- Exact 0.90 probability interval in $S_{I n+l}=$ shaded intervals in panel B $=\left[S_{I \mathrm{a}}, S_{\mathrm{lb}}\right]$ and $\left[S_{I \mathrm{c}}, S_{I 100}\right]$, where a, b and c are percentile values of $S_{I \mathrm{a}}, S_{\mathrm{lb}}$ and $S_{\mathrm{Ic}}$, and

$$
\begin{aligned}
& \operatorname{pdf}\left(S_{I \mathrm{a}}\right)=\operatorname{pdf}\left(S_{I \mathrm{~b}}\right)=\operatorname{pdf}\left(S_{I \mathrm{c}}\right) \\
& \operatorname{cdf}\left(S_{I k}\right)=k \quad \text { for } k=\mathrm{a}, \mathrm{b}, \mathrm{c} \\
& (100-\mathrm{c})+(\mathrm{b}-\mathrm{a})=90
\end{aligned}
$$

- Exact 0.90 probability interval in $B G_{n+1}=$ shaded intervals in panel $\mathrm{D} \neq$ $\left[f\left(S_{I 100}\right), f\left(S_{I c}\right)\right]$ and $\left[f\left(S_{I b}, f\left(S_{I a}\right)\right]\right.$, or

$$
\operatorname{pdf}\left(f\left(S_{\text {Ia }}\right)\right) \neq \operatorname{pdf}\left(f\left(S_{I \mathrm{~b}}\right)\right) \neq \operatorname{pdf}\left(f\left(S_{\text {Ic }}\right)\right)
$$

Consequently, to obtain the exact probability intervals in $B G_{n+1}$, Monte Carlo simulations using a minimum of $10,000 S_{I n+l}$ values needs to be done to achieve an 
accuracy of $1 \%$. While such a Monte Carlo simulation can provide the appropriate probability density in $B G_{n+1}$, it is too computationally expensive to generate useful and effective probability intervals quickly enough for clinical decision support.

\subsubsection{Clinical feasibility}

An estimated computational comparison between calculating the equal-tailed and the exact probability intervals is summarised in Table 2 . The computational timeframe for calculating the exact probability interval is clearly not currently feasible for this type of real-time clinical control. In conclusion, assuming equal-tailed probability intervals provides fast, clinically viable and slightly conservative estimates for the most likely ranges of $B G_{n+l}$. Therefore, it should not compromise patient safety when used to assist clinical decision making. As the example in Figure 5 shows, the 0.90 equaltailed probability interval in $B G_{n+1}([1.9,5.4])$ covers most of the exact 0.90 most probable range $([1.8,4.5]$ and $[4.7,4.9])$, providing an effective and clinically useful estimate with far less effort.

\subsection{Cross Validation}

The stochastic $S_{I}$ model can be integrated into the system model of Equations (1)-(5) for use in control. This step allows the blood glucose level probability intervals one hour following an insulin and/or nutrition intervention to be found directly based on the probability density function of $S_{I n+1}$. Cross validation is used in this study to test the generality of the SPRINT cohort and resulting stochastic model.

For cross validation, the 165 patients are divided into 5 random groups. Each group has comparable medical conditions, sex, age, and APACHE (Acute Physiology and 
Chronic Health Evaluation) scores. Five stochastic $S_{I}$ models are then built, each one using fitted $S_{I}$ data from 4 out of 5 groups. Each stochastic model is then evaluated against the group that is not used for creating the model. Thus, a stochastic model created from Groups $1-4$ is evaluated against a patient in Group 5 in the following steps:

1. At hour 0 , the patient's identified $S_{I}$ is $S_{I 0}$. The stochastic model then produces the $5^{\text {th }}, 25^{\text {th }}, 75^{\text {th }}$ and $95^{\text {th }}$ percentiles for probable $S_{I}$ at hour 1 , denote $S_{I 1-5}$, $S_{I 1-25}, S_{I 1-75}$ and $S_{I 1-95}$

2. Examine if the identified $S_{I I}$ is within the inter-quartile probability interval between $S_{I-25}$ and $S_{I}$ 1-75, and the equal-tailed 0.90 probability interval between $S_{I 1-5}$ and $S_{I 1-95}$.

3. Repeat the process from hour 1 until the end of the patient data.

\subsection{Further model applications: virtual patients and trial simulations}

A generic stochastic model is built using all 165 SPRINT patients. Incorporating this stochastic model into the glucose-insulin system model presented in Equations (1)-(5), typical critical care patient dynamics can be reproduced numerically given initial conditions in $G, Q$ and $I$, starting dextrose and insulin inputs, and a starting $S_{I}$ value.

\subsubsection{Virtual patient generation and trial implementation}

To generate a "virtual patient" cohort similar to the SPRINT cohort, and therefore representative of a critically ill population, initial conditions to Equations (1)-(5) are generated to approximate the statistical distribution of these parameters, as recorded from the SPRINT cohort. Initial $S_{I}$ values are randomly selected from the initial 
values fitted from the SPRINT $S_{I}$ data. Trial lengths were also randomly generated to create a similar statistical distribution to that in the SPRINT trials. Profiles of $S_{I}$ are then generated using the stochastic model. Using these profiles in Equations (1)-(5), "virtual patients" are created that (statistically) approximate the SPRINT cohort on which the model was created, providing a platform for clinical trial simulation and control development. A virtual control trial simulation consists of hourly cycles of the following steps:

1. Generate an hourly $S_{I n+l}$ value from the stochastic model defined probability density function of the previous hourly $S_{I}$ value $S_{I n}$.

2. Generate the end-of-hour virtual blood glucose level using the generated $S_{I n+1}$ and the specified control (insulin and nutrition) interventions at hour $n+1$ in Equations (1)-(5). Standard Glucocard ${ }^{\mathrm{TM}}$ blood glucose measurement error [36] is numerically added to the generated blood glucose level, matching the clinical conditions in Christchurch Hospital ICU [12, 37].

3. A new $S_{I}$, denoted $S_{I}$, is fit to the blood glucose levels including random noise that are "measured" during hour $n+1$. Integral-based parameter identification is used to identify $S_{I}{ }^{\prime}[12,15]$.

4. The median and equal-tailed 0.90 and inter-quartile probability intervals of potential $S_{I}$ are generated from the identified $S_{I}$ obtained from step 3, using the value of $S_{I}$ ' in the stochastic model as described in Figure 4.

5. Interventions are determined that position the median glucose and probability intervals to criterion defined by the control algorithm.

6. Return to step 1 with $S_{I n+l}$ being the new $S_{I n}$. 
Essentially, a virtual patient's $S_{I}$ evolution follows the joint probability density contour in Figure 1, making its way to the highest probability density regions. Each $S_{I n+l}$ is dependent on the previous state, $S_{I n}$, where the probability density function of of $S_{I n+I}$ is defined in Equations (10)-(11). Hence, in step 1 above, $S_{I}$ takes a walk to a point, say $y$, along the probability density function curve $p\left(S_{I n+l}=y \mid S_{I n}=x\right)$ as shown in Figure 4. Step 6 then "walk" the $S_{I}$ across (along $S_{I n+1}$ axis) to where $S_{I n}=y$, and the process repeats.

\subsubsection{Virtual trial control algorithm}

The control algorithm used in this study is a target-shooting algorithm that probabilistically minimises the risk of hypoglycemia. Interventions include insulin bolus injections, and insulin and dextrose infusions. The algorithm obeys the following rules in a prioritised order.

1. The lower bound of the equal-tailed 0.90 probability intervals in blood glucose levels resulted from control interventions must never be lower than $4 \mathrm{mmol} / \mathrm{L}$.

2. The controller specified dextrose feeding rate must be greater than or equal to the patient specific minimum $[6,12]$.

3. The total hourly insulin input must not exceed $6 \mathrm{U}[10,38]$.

4. Saturation in $Q$ (Equations (1)-(2)) must not exceed $200 \mathrm{mU} / \mathrm{L} / \mathrm{min}$ :

$$
Q-\frac{Q}{1+\alpha_{G} Q} \leq 200 \mathrm{~mL} / \mathrm{L} / \mathrm{min}
$$

5. The target blood glucose level (median of the blood glucose probability density function) is $85 \%$ of the blood glucose level at the time of intervention, to a minimum of $4.5 \mathrm{mmol} / \mathrm{L}$.

6a. To lower blood glucose levels, the control algorithm seeks to lower the dextrose feeding rate before adding insulin in bolus (injection) form. Insulin 
infusions may be used to lower blood glucose levels without resulting in excess interstitial insulin saturation (Rule 4).

6b. To increase low blood glucose levels, the control algorithm reduces insulin inputs first, and then increases dextrose rates.

The first 4 rules are designed for patient safety. If the target blood glucose level cannot be achieved without violating these first 4 rules, it is "bounded" so it can be achieved within these limitations. The final 3 rules define how the algorithm prioritises achieving the target blood glucose level.

\section{Results and Discussion}

\subsection{Cross validation}

Table 3 shows the general information on the 5 patient groups used in cross validation.

The percentage of fitted $S_{I}$ within the equal-tailed 0.90 and inter-quartile probability intervals for the group not used in creating the cross validation model is summarised in Table 4. Each group produced similar results, which were also comparable to the overall result. Thus, there is no significant difference between the 5 stochastic models created and the SPRINT cohort can be considered a generic representation of this ICU population.

More specifically, the mean per patient average of identified $S_{I}$ values within the equal-tailed probability interval is $86.6 \%$, and $54.0 \%$ for the inter-quartile interval. The equal tailed 0.90 probability interval slightly underestimates the identified $S_{I}$ 
range, because when $S_{I n}$ is close to its fitting constraints, the $S_{I n+l}$ probability density function tends to peak near the boundary value. This behaviour causes some regions outside of the equal-tailed 0.90 probability intervals to have higher probability density than some regions within the interval, as shown in Figures 3 and 5. Thus, the compromise of using equal-tailed probability intervals has negligible impact compared to the computational gain over calculating the exact blood glucose ranges of the highest 0.90 probability density.

The inter-quartile probability intervals include slightly over $50 \%$ of fitted $S_{I}$, suggesting that most of the time the higher probability density is concentrated about the median of the probability density functions, as also seen in Figures 3 and 5. Similarly, the 0.90 probability interval has slightly less than $90 \%$ of all measurements, suggesting that there are slightly more outliers than represented in the model. Both results suggest that the assumptions used are slightly inexact, but within typical variations caused by the $7-12 \%$ measurement error $[36,39]$.

\subsection{Virtual control trial results}

A virtual cohort of 200 patients was created and tested in simulated trials. Blood glucose probability intervals from control inputs were produced with each control intervention using the stochastic model. Hourly blood glucose measurements including normally distributed random measurement noise were analysed against the probability intervals. An example of a virtual trial is shown in Figure 6. The top panel shows the blood glucose excursion through time. The bold crosses are the virtually generated blood glucose levels at one hour intervals, with thick bars indicating the standard 7\% Glucocard ${ }^{\mathrm{TM}}$ measurement error [36]. Hourly $S_{I}$ is shown in the bottom 
panel. Control interventions are determined hourly and shown in the middle panel, producing stochastic model derived probability median values and intervals in blood glucose shown as circles with thin bars in the top panel.

The average fractions of virtual trial blood glucose levels within the equal-tailed 0.90 and inter-quartile probability intervals for each patient are shown in Figure 7. The logistic mean of percentage blood glucose levels within the equal-tailed 0.90 and inter-quartile probability interval are $87.0 \%$ and $45.7 \%$ respectively. These results are in general agreement with the cross validation results in Table 5. These per patient results also show the overall validity in total, as well as over all virtual patients, with very few outlying virtual patients.

A few trials in Figure 7 have comparatively lower percentages within the probability intervals. These trials are all shorter, and consequently a small number of blood glucose levels outside of the probability intervals are reported as higher percentages of the total. Figure 8 illustrates how shorter trials can produce outliers, where most variability is gone after 50-75 hours, indicating stabilised patient condition under long term, tight glycemic control with little variability in glycemic level.It also shows how as time length of trial increases, the 0.90 and inter-quartile probability intervals are both slightly conservative estimates by $2-4 \%$, as also seen in the median values of Figure 7.

The control algorithm used to run these virtual trials targets the blood glucose probability density medians to the desired level. The controller specified interventions should thus theoretically result in the median blood glucose level being at the desired 
level one hour later. The distribution of virtual trial deviations (percentage value) from the probability density medians is shown in Figure 9. The mean per patient average deviation is $8.84 \%$, as compared to a normally distributed random measurement error of $7-10 \%$. Outliers are again associated with shorter trial length.

Examining the signed blood glucose deviations from probabilistic medians, 58\% are negative. This result suggests that slightly higher density may be concentrated at higher $S_{I}$ in the probability density functions. In future, this blood glucose deviation could be "corrected" for in the control algorithm by targeting a percentile higher than the $50^{\text {th }}$, for example the $58^{\text {th }}$ percentile, until the deviation is minimised, thus further improving the targeting accuracy. Similar manipulation may also be carried out to "correct" the accuracy of the probability intervals.

Virtual trial controller performance is compared to the actual clinical SPRINT results and summarised in Table 5. The blood glucose is more tightly controlled, with a lower mean level (5.5 v.s $5.9 \mathrm{mmol} / \mathrm{L})$, and a similar, but safer, $5-95^{\text {th }}$ percentile interval $([4.1-8.3]$ v.s $[3.6-7.8])$. The percentage of blood glucose in the $4-6 \mathrm{mmol} / \mathrm{L}$ range increases from $61 \%$ to $72 \%$. Without the stochastic targeting, the effectively same control protocol delivered only $64 \%$ time in this band [6]. It is a significant difference as tighter control has been shown to imply a better mortality outcome [4]. Again, the control quality is linked with trial length. Shorter trials tend to give a sparser blood glucose distribution and higher variance. This was also seen in SPRINT where the cohort with length of stay $<3$ days had greater variability. 
Finally, a similar comparison of is shown in Figure 10. The percentage lower than 4 $\mathrm{mmol} / \mathrm{L}$ is slightly increased from $3.3 \%$ to $4.5 \%$, with $0 \%$ under $2.5 \mathrm{mmol} / \mathrm{L}$. The percentage lower than $4 \mathrm{mmol} / \mathrm{L}$ can be easily reduced by setting a higher percentile limit in Rule 1, and/or increasing the minimum target from $4.5 \mathrm{mmol} / \mathrm{L}$ to $4.8-5.0$ $\mathrm{mmol} / \mathrm{L}$ in Rule 5.

\section{Conclusions}

The stochastic model presented defines the probability density functions of blood glucose levels one hour following a known glycemic control intervention, and thus enables more knowledgeable and accurate prediction for glycemic control. The model created from 165 SPRINT trial patients was cross validated, verifying the generality of the chosen cohort and the method used to create the stochastic model. The model was then used to create 200 virtual patients representative of hyperglycaemic ICU patients based on clinical SPRINT protocol results. A stochastically targeted control protocol was then tested through numerical simulations.

Both the cross validation and the virtual trial simulation results confirm that the stochastic model provides accurate probabilistic knowledge. The cross validation results show that the 0.90 and inter-quartile probability intervals provide a slightly conservative bound, but accurately capture the cohort. The virtual trials also show that a stochastically-driven computerised control method can outperform a simplified version of the same basic insulin and nutrition control protocol, demonstrating further benefits of this model and/or computer control in general. In summary, the stochastic glucose-insulin system model presented can effectively capture critical care patient 
behaviour, enhance glycemic control and create a virtual patient platform for further development.

\section{References}

1. Chase, J., G.M. Shaw, X.W. Wong, T. Lotz, J. Lin, and C.E. Hann, Modelbased Glycaemic Control in Critical Care - A review of the state of the possible. Biomedical Signal Processing and Control, 2006. 1(1): p. 3-21.

2. Krinsley, J.S., Association between hyperglycemia and increased hospital mortality in a heterogeneous population of critically ill patients. Mayo Clin Proc, 2003. 78(12): p. 1471-1478.

3. Van den Berghe, G., P. Wouters, F. Weekers, C. Verwaest, F. Bruyninckx, M. Schetz, D. Vlasselaers, P. Ferdinande, P. Lauwers, and R. Bouillon, Intensive insulin therapy in the critically ill patients. N Engl J Med, 2001. 345(19): p. 1359-1367.

4. Chase, J.G., C.E. Hann, G.M. Shaw, X.W. Wong, J. Lin, T. Lotz, A.J. Le Compte, and T. Lonergan, An Overview of Glycemic Control in Critical Care - Relating Performance and Clinical Results. Journal of Diabetes Science and Technology (http://www.journalofdst.org), 2006. 1(1): p. 82-91.

5. Turina, M., M. Christ-Crain, and H.C. Polk, Jr., Diabetes and hyperglycemia: strict glycemic control. Crit Care Med, 2006. 34(9 Suppl): p. S291-300.

6. Lonergan, T., A. LeCompte, M. Willacy, J.G. Chase, G.M. Shaw, X.W. Wong, T. Lotz, J. Lin, and C.E. Hann, A Simple Insulin-Nutrition Protocol for Tight Glycemic Control in Critical Illness: Development and Protocol Comparison. Diabetes Technol Ther, 2006. 8(2): p. 191-206.

7. Lonergan, T., A. LeCompte, M. Willacy, J. Chase, G. Shaw, C. Hann, T. Lotz, J. Lin, and X. Wong, A Pilot Study of the SPRINT Protocol for Tight Glycaemic Control in Critically Ill Patients. Diabetes Technol Ther, 2006. 84(4): p. 449-462.

8. Plank, J., J. Blaha, J. Cordingley, M.E. Wilinska, L.J. Chassin, C. Morgan, S. Squire, M. Haluzik, J. Kremen, S. Svacina, W. Toller, A. Plasnik, M. Ellmerer, R. Hovorka, and T.R. Pieber, Multicentric, randomized, controlled trial to evaluate blood glucose control by the model predictive control algorithm versus routine glucose management protocols in intensive care unit patients. Diabetes Care, 2006. 29(2): p. 271-6.

9. Chee, F., T.L. Fernando, A.V. Savkin, and V. van Heeden, Expert PID control system for blood glucose control in critically ill patients. IEEE Trans Inf Technol Biomed, 2003. 7(4): p. 419-25. 
10. Chase, J.G., G.M. Shaw, J. Lin, C.V. Doran, C. Hann, M.B. Robertson, P.M. Browne, T. Lotz, G.C. Wake, and B. Broughton, Adaptive bolus-based targeted glucose regulation of hyperglycaemia in critical care. Med Eng Phys, 2005. 27(1): p. 1-11.

11. Chase, J., G. Shaw, A. LeCompte, D. Lee, T. Lonergan, M. Willacy, X. Wong, J. Lin, T. Lotz, and C. Hann. Tight Glycaemic Control in Critical Care Using Insulin and Nutrition - the SPRINT Protocol. in 6th Annual Diabetes Technology Society Meeting. 2006. Atlanta, GA: Diabetes Technology Society.

12. Wong, X.W., I. Singh-Levett, L.J. Hollingsworth, G.M. Shaw, C.E. Hann, T. Lotz, J. Lin, O.S. Wong, and J.G. Chase, A novel, model-based insulin and nutrition delivery controller for glycemic regulation in critically ill patients. Diabetes Technol Ther, 2006. 8(2): p. 174-90.

13. Van den Berghe, G., A. Wilmer, G. Hermans, W. Meersseman, P.J. Wouters, I. Milants, E. Van Wijngaerden, H. Bobbaers, and R. Bouillon, Intensive Insulin Therapy in the Medical ICU. N Engl J Med, 2006. 354(5): p. 449-61.

14. Lonergan, T., A.L. Compte, M. Willacy, J.G. Chase, G.M. Shaw, C.E. Hann, T. Lotz, J. Lin, and X.W. Wong, A pilot study of the SPRINT protocol for tight glycemic control in critically Ill patients. Diabetes Technol Ther, 2006. 8(4): p. 449-62.

15. Hann, C.E., J.G. Chase, J. Lin, T. Lotz, C.V. Doran, and G.M. Shaw, Integralbased parameter identification for long-term dynamic verification of a glucose-insulin system model. Comput Methods Programs Biomed, 2005. 77(3): p. 259-270.

16. Hann, C., J. Chase, and G. Shaw, Integral-based Identification of Patient Specific Parameters for a Minimal Cardiac Model. Computer Methods and Programs in Biomedicine, 2006. 81(2): p. 181-192.

17. Lotz, T.F., J.G. Chase, K.A. McAuley, G.M. Shaw, C.E. Hann, and J.I. Mann. A highly correlated method to assess insulin resistance in broad populations. in 12th International Conf on Biomedical Engineering (ICBME). 2005. Singapore.

18. Lotz, T., J. Chase, K. McAuley, J. Lin, X. Wong, C. Hann, and S. Andreassen. Integral-Based Identification of a Physiological Insulin and Glucose Model on Euglycaemic Clamp Trials. in 14th IFAC Symposium on System Identification (SYSID 2006). 2006. Newcastle, Australia.

19. Lotz, T., J. Chase, K. McAuley, D. Lee, J. Lin, C. Hann, and J.I. Mann, Transient and steady state euglycemic clamp validation of a model for glycemic control and insulin sensitivity testing. Diabetes Technol Ther, 2006. 8(3): p. 338-346. 
20. Carson, E.R. and C. Cobelli, Modelling methodology for physiology and medicine. Academic Press Series in Biomedical Engineering. 2001, San Diego: Academic Press. xiv, 421.

21. Lin, J., Lee, DS, Chase, JG, Hann, CE, Lotz, T and Wong, XW, Stochastic Modelling of Insulin Sensitivity Variability in Critical Care. Biomedical Signal Processing \& Control, 2006. 1: p. 229-242.

22. Natali, A., A. Gastaldelli, S. Camastra, A.M. Sironi, E. Toschi, A. Masoni, E. Ferrannini, and A. Mari, Dose-response characteristics of insulin action on glucose metabolism: a non-steady-state approach. Am J Physiol Endocrinol Metab, 2000. 278(5): p. E794-801.

23. Prigeon, R.L., M.E. Roder, D. Porte, Jr., and S.E. Kahn, The effect of insulin dose on the measurement of insulin sensitivity by the minimal model technique. Evidence for saturable insulin transport in humans. J Clin Invest, 1996. 97(2): p. 501-7.

24. Scott, D.W., Multivariate density estimation: theory, practice, and visualization. Wiley series in probability and mathematical statistics. 1992, New York: John Wiley \& sons, Inc.

25. Ferrannini, E. and C. Cobelli, The kinetics of insulin in man. II. Role of the liver. Diabetes Metab Rev, 1987. 3(2): p. 365-97.

26. Sherwin, R.S., K.J. Kramer, J.D. Tobin, P.A. Insel, J.E. Liljenquist, M. Berman, and R. Andres, A model of the kinetics of insulin in man. J Clin Invest, 1974. 53(5): p. 1481-92.

27. Ferrannini, E., A. Gastaldelli, Y. Miyazaki, M. Matsuda, A. Mari, and R.A. DeFronzo, beta-Cell function in subjects spanning the range from normal glucose tolerance to overt diabetes: a new analysis. J Clin Endocrinol Metab, 2005. 90(1): p. 493-500.

28. Van Cauter, E., F. Mestrez, J. Sturis, and K.S. Polonsky, Estimation of insulin secretion rates from C-peptide levels. Comparison of individual and standard kinetic parameters for C-peptide clearance. Diabetes, 1992. 41(3): p. 368-77.

29. Ellemann, K., B. Thorsteinsson, S. Fugleberg, B. Feldt-Rasmussen, O.O. Andersen, P. Gronbaek, and C. Binder, Kinetics of insulin disappearance from plasma in cortisone-treated normal subjects. Clin Endocrinol (Oxf), 1987. 26(5): p. 623-628.

30. Thorell, A., O. Rooyackers, P. Myrenfors, M. Soop, J. Nygren, and O.H. Ljungqvist, Intensive insulin treatment in critically ill trauma patients normalizes glucose by reducing endogenous glucose production. J Clin Endocrinol Metab, 2004. 89(11): p. 5382-6.

31. DeFronzo, R.A., J.D. Tobin, and R. Andres, Glucose clamp technique: a method for quantifying insulin secretion and resistance. Am J Physiol, 1979. 237(3): p. E214-23. 
32. Insel, P.A., J.E. Liljenquist, J.D. Tobin, R.S. Sherwin, P. Watkins, R. Andres, and M. Berman, Insulin control of glucose metabolism in man. J Clin Invest, 1975. 55(5): p. 1057-66.

33. Transberg, K., P. Hagander, and J. Thorell, Disappearance of insulin in man: variation with the plasma insulin level. Acta Endocrinol (Copenh), 1981. 97: p. 391-397.

34. McCowen, K.C., A. Malhotra, and B.R. Bistrian, Stress-induced hyperglycemia. Crit Care Clin, 2001. 17(1): p. 107-124.

35. Casella, G. and R.L. Berger, Distributions of Functions of a Random Variable, in Statistical Inference. 2002, Duxbury. p. 47-54.

36. Arkray, Glucocard ${ }^{T M}$ Test Strip 2 Data Sheet. 2001, Arkray Inc.: Japan.

37. Wong, X., J.G. Chase, G.M. Shaw, C. Hann, T. Lotz, J. Lin, I. Singh-Levett, L. Hollingsworth, O. Wong, and S. Andreassen, Model Predictive Glycaemic Regulation in Critical Illness using Insulin and Nutrition Input: a Pilot Study. Medical Engineering \& Physics, 2006. 28(7): p. 665-681.

38. Chase, J.G., G.M. Shaw, J. Lin, C.V. Doran, M. Bloomfield, G.C. Wake, B. Broughton, C. Hann, and T. Lotz, Impact of Insulin-Stimulated Glucose Removal Saturation on Dynamic Modelling and Control of Hyperglycaemia. International Journal of Intelligent Systems Technologies and Applications (IJISTA), 2004. 1(1/2): p. 79-94.

39. Lotz, T., J.G. Chase, K. McAuley, G. Shaw, X.-W. Wong, J. Lin, A. Le Compte, C.E. Hann, and J.I. Mann. Highly Correlated Model-Based Testing of Insulin Sensitivity - Initial Results for a Proposed Low-Intensity Test. in 6th Annual Diabetes Technology Society Meeting. 2006. Atlanta, USA. 
Table 1. Comparison of clinical SPRINT and retrospective cohorts.

\begin{tabular}{ccc} 
& All & $\begin{array}{c}\text { Length of stay }>= \\
\text { 3 days }\end{array}$ \\
\hline \hline $\begin{array}{c}\text { Number of } \\
\text { patients }\end{array}$ & 165 & 120 \\
$\begin{array}{c}\text { Age (years) } \\
\% \text { Male }\end{array}$ & $65[50-74]$ & $65[49-74]$ \\
$\begin{array}{c}\text { APACHE II } \\
\text { score }\end{array}$ & $19[15-25]$ & $19[15-26]$ \\
$\begin{array}{c}\text { APACHE II } \\
\text { risk of death }\end{array}$ & $29 \%[15-55 \%]$ & $31 \%[16-56 \%]$ \\
\hline
\end{tabular}

* Only $74 \%$ of APACHE II data for the retrospective cohort was available. Data are expressed as median [interquartile range] where appropriate. 
Table 2. Comparison between probability interval computational cost.

\begin{tabular}{|c|c|c|c|c|}
\hline $\begin{array}{c}0.90 \\
\text { probability } \\
\text { interval } \\
\text { calculation } \\
\end{array}$ & Equal-tailed & $\begin{array}{c}\text { Calculation } \\
\text { Time }\end{array}$ & Exact & $\begin{array}{c}\begin{array}{c}\text { Calculation } \\
\text { Time }\end{array} \\
\end{array}$ \\
\hline \multirow[t]{3}{*}{ Steps } & $\begin{array}{l}\text { 1. Calculate the } 5^{\text {th }} \text { and } \\
95^{\text {th }} \text { percentiles in } S_{1 n+1} \text {, } \\
S_{15} \text { and } S_{195}\end{array}$ & $\sim 0 \mathrm{sec}$ & $\begin{array}{l}\text { 1. Generate } 10,000 S_{\mid n+1} \\
\text { using the derived pdf } \\
\text { from the stochastic } \\
\text { model }\end{array}$ & $2 \mathrm{sec}$ \\
\hline & $\begin{array}{l}\text { 2. Equal-tailed } 0.90 \\
\text { probability interval in } \\
B G_{n+1}=\left[f\left(S_{195}\right), f\left(S_{15}\right)\right]\end{array}$ & $1 \mathrm{sec}$ & $\begin{array}{l}\text { 2. Calculate } B G_{n+1} \text { for } \\
\text { each of the } 10,000 \\
S_{I n+1} \text { values }\end{array}$ & $\begin{array}{c}5,000 \mathrm{sec}= \\
83 \mathrm{~min}\end{array}$ \\
\hline & & & $\begin{array}{l}\text { 3. Sort the } 10,000 B G_{n+1} \\
\text { values and find the } 5^{\text {th }} \\
\text { and } 95^{\text {th }} \text { percentiles }\end{array}$ & $1 \mathrm{sec}$ \\
\hline Total Time & & $\sim 1 \mathrm{sec}$ & & $\sim 83 \mathrm{~min}$ \\
\hline
\end{tabular}


Table 3. Sub-cohorts of 165 SPRINT patients for cross validation.

\begin{tabular}{c|c|c|c|c|c|c} 
Group & $\begin{array}{c}\text { no. of } \\
\text { patients }\end{array}$ & age $(\mathrm{yr})$ & $\begin{array}{c}\text { gender } \\
(\% \text { male })\end{array}$ & $\begin{array}{c}\text { average blood } \\
\text { glucose level } \\
(\mathrm{mmol} / \mathrm{L})\end{array}$ & $\begin{array}{c}\text { length of trial } \\
(\mathrm{Hr})\end{array}$ & $\begin{array}{c}\text { time in 4- } \\
6.1 \mathrm{mmol} / \mathrm{L} \\
\text { band }(\%)\end{array}$ \\
\hline \hline $\mathbf{1}$ & 33 & $57.4(19.4)$ & $69.7 \%$ & $6.2(1.9)$ & $144.6(189.1)$ & $58.1(24.5)$ \\
$\mathbf{2}$ & 33 & $66.5(13.8)$ & $63.6 \%$ & $5.9(0.7)$ & $141.1(167.7)$ & $59.0(17.3)$ \\
$\mathbf{3}$ & 33 & $61.0(15.6)$ & $63.6 \%$ & $6.0(0.9)$ & $138.6(169.4)$ & $59.8(22.1)$ \\
$\mathbf{4}$ & 33 & $63.7(15.9)$ & $60.6 \%$ & $6.0(0.7)$ & $142.7(143.2)$ & $58.2(16.1)$ \\
$\mathbf{5}$ & 33 & $56.5(18.1)$ & $72.7 \%$ & $5.9(0.8)$ & $139.8(169.0)$ & $59.1(21.0)$ \\
overall & 165 & $61.0(16.5)$ & $66.1 \%$ & $6.0(1.0)$ & $141.4(167.7)$ & $58.9(20.2)$
\end{tabular}

\begin{tabular}{c|c|c|c|c|c} 
Group & $\begin{array}{c}\text { average } \\
\text { insulin usage } \\
\text { (U/Hr) }\end{array}$ & $\begin{array}{c}\text { average } \\
\text { absolute goal } \\
\text { feed [ml/hr] }\end{array}$ & $\begin{array}{c}\text { average } \% \\
\text { goal feed }\end{array}$ & $\begin{array}{c}\text { mortality } \\
(\%)\end{array}$ & APACHE II \\
\hline $\mathbf{1}$ & $2.6(1.1)$ & $75.3(10.6)$ & $48.3(38.7)$ & $72.7 \%$ & $20.5(8.8)$ \\
$\mathbf{2}$ & $2.6(1.2)$ & $73.0(10.3)$ & $52.0(36.0)$ & $81.8 \%$ & $17.7(7.1)$ \\
$\mathbf{3}$ & $2.6(0.7)$ & $74.7(10.7)$ & $52.5(42.3)$ & $84.8 \%$ & $20.9(8.8)$ \\
$\mathbf{4}$ & $2.7(1.0)$ & $70.9(10.3)$ & $54.0(32.7)$ & $87.9 \%$ & $21.3(6.4)$ \\
$\mathbf{5}$ & $2.5(0.7)$ & $77.3(10.4)$ & $52.7(32.5)$ & $82.4 \%$ & $20.5(7.3)$ \\
overall & $2.6(0.9)$ & $74.2(10.5)$ & $51.9(36.4)$ & $81.9 \%$ & $20.2(7.7)$
\end{tabular}


Table 4. Stochastic insulin sensitivity model cross validation results.

\begin{tabular}{cccc}
\hline Group & $\begin{array}{c}\text { Groups used in } \\
\text { creating } \\
\text { stochastic model }\end{array}$ & $\begin{array}{c}\text { Average percentage of } \\
\text { fitted } S \text {, within equal-tailed } \\
0.90 \text { probability interval }\end{array}$ & $\begin{array}{c}\text { Average percentage of fitted } \\
\text { S, within inter-quartile } \\
\text { probability interval }\end{array}$ \\
\hline \hline 1 & {$[-, 2,3,4,5]$} & $85.7 \%$ & $54.2 \%$ \\
2 & {$[1,-, 3,4,5]$} & $83.2 \%$ & $52.3 \%$ \\
3 & {$[1,2,-, 4,5]$} & $87.4 \%$ & $54.7 \%$ \\
4 & {$[1,2,3,-, 5]$} & $88.7 \%$ & $54.1 \%$ \\
5 & {$[1,2,3,4,-]$} & $87.9 \%$ & $54.8 \%$ \\
overall & {$[1,2,3,4,5]$} & $86.6 \%$ & $54.0 \%$ \\
\hline
\end{tabular}


Table 5. Virtual trial results compared to clinical SPRINT results.

\begin{tabular}{|c|c|c|c|}
\hline \multirow{2}{*}{\multicolumn{4}{|c|}{ Virtual Trials }} \\
\hline & & & \\
\hline Number of patients & 165 & 200 & \multirow{3}{*}{ hours } \\
\hline Hours of control & 23,324 & 33,889 & \\
\hline Total BG measurements & 15,874 & 34,089 & \\
\hline BG mean a & $5.9[4.1-8.3]$ & $5.5[3.6-7.8]$ & \multirow{7}{*}{$\begin{array}{l}\mathrm{mmol} / \mathrm{L} \\
\mathrm{mmol} / \mathrm{L}\end{array}$} \\
\hline $\mathrm{BG}$ standard deviation ${ }^{a}$ & 1.3 & 1.3 & \\
\hline Percentage between $4-6.1 \mathrm{mmol} / \mathrm{L}$ & $61 \%$ & $72 \%$ & \\
\hline Percentage between $4-7.0 \mathrm{mmol} / \mathrm{L}$ & $82 \%$ & $82 \%$ & \\
\hline Percentage between $4-7.75 \mathrm{mmol} / \mathrm{L}$ & $89 \%$ & $87 \%$ & \\
\hline Percentage $<4 \mathrm{mmol} / \mathrm{L}$ & $3.3 \%$ & $4.5 \%$ & \\
\hline Percentage $<2.5 \mathrm{mmol} / \mathrm{L}$ & $0.1 \%$ & $0.02 \%$ & \\
\hline \multicolumn{4}{|l|}{ Per-patient data } \\
\hline Hours of control & 95 [12 - 447] & 79 [20 - 688] & \multirow{7}{*}{$\begin{array}{l}\mathrm{mmol} / \mathrm{L} \\
\mathrm{mmol} / \mathrm{L} \\
\mathrm{U} \\
\mathrm{ml} / \mathrm{hr} \\
\mathrm{kCal} / \mathrm{dav}\end{array}$} \\
\hline Number of measurements & $68[10-271]$ & $80[21-689]$ & \\
\hline BG mean & $5.9[5.0-7.4]$ & $5.3[4.6-7.5]$ & \\
\hline BG standard deviation & $1.1[0.7-2.3]$ & $1.0[0.5-2.1]$ & \\
\hline Median hourly insulin & $2.5[1.3-4.1]$ & $3.2[1.3-4.4]$ & \\
\hline Median nutrition rate (RESOURCE Diabetic) & $37.5[0-80.3]$ & $28.2[18.1-42.9]$ & \\
\hline (assuming $1.06 \mathrm{cal} / \mathrm{ml}$ ) & 954 [0 - 2043] & 717 [461 - 1093] & \\
\hline Median percentage of goal feed & $52.7 \%$ & $38.4 \%$ & \\
\hline
\end{tabular}

${ }^{a}$ Lognormal distribution

Data are expressed as median [5th-95th percentile range] as appropriate 


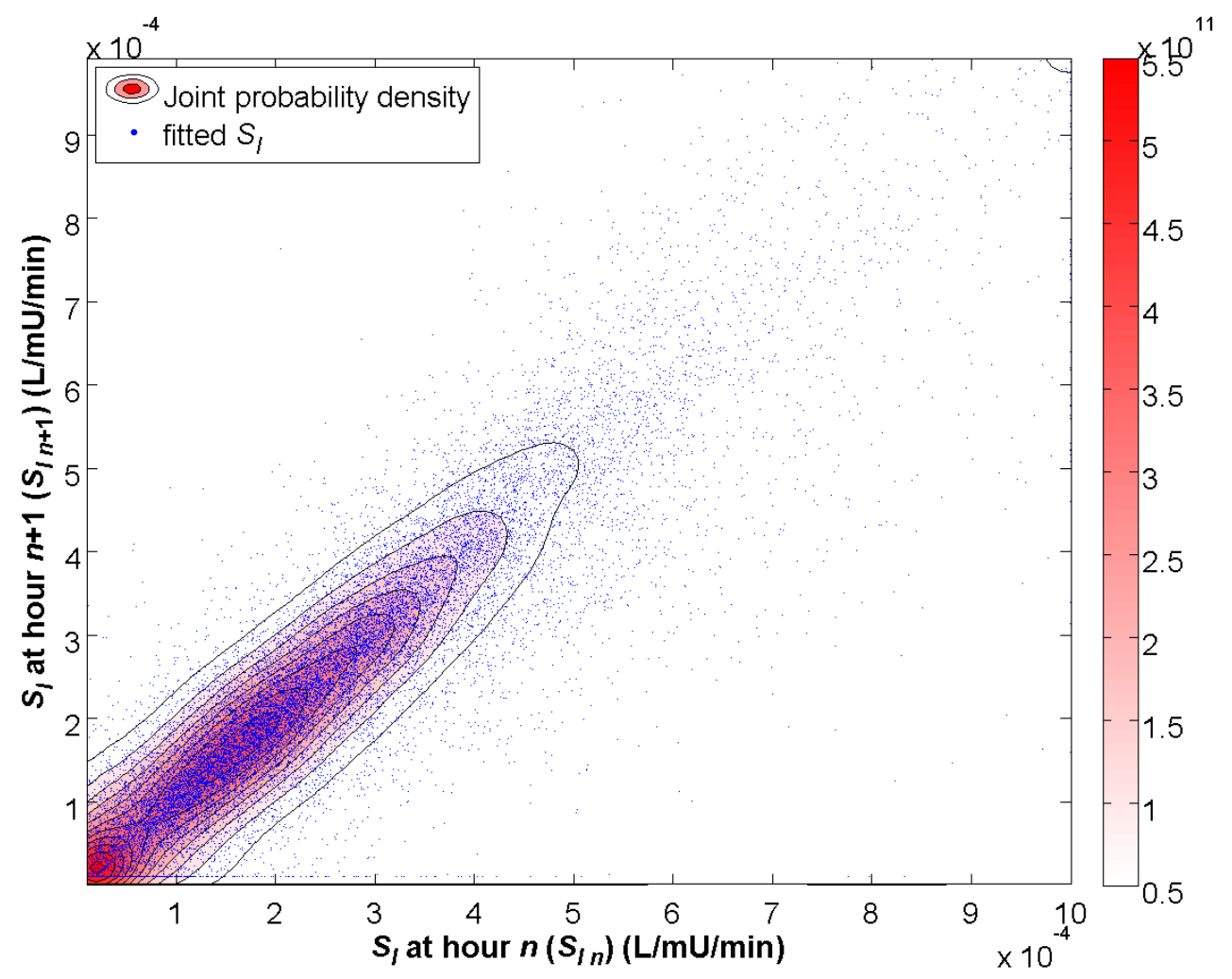

Figure 1. Fitted hourly $S_{I}$ variation (dots) and joint kernel probability density (shaded contours). The area under the joint probability density function surface in the model space sums to 1.0 . 


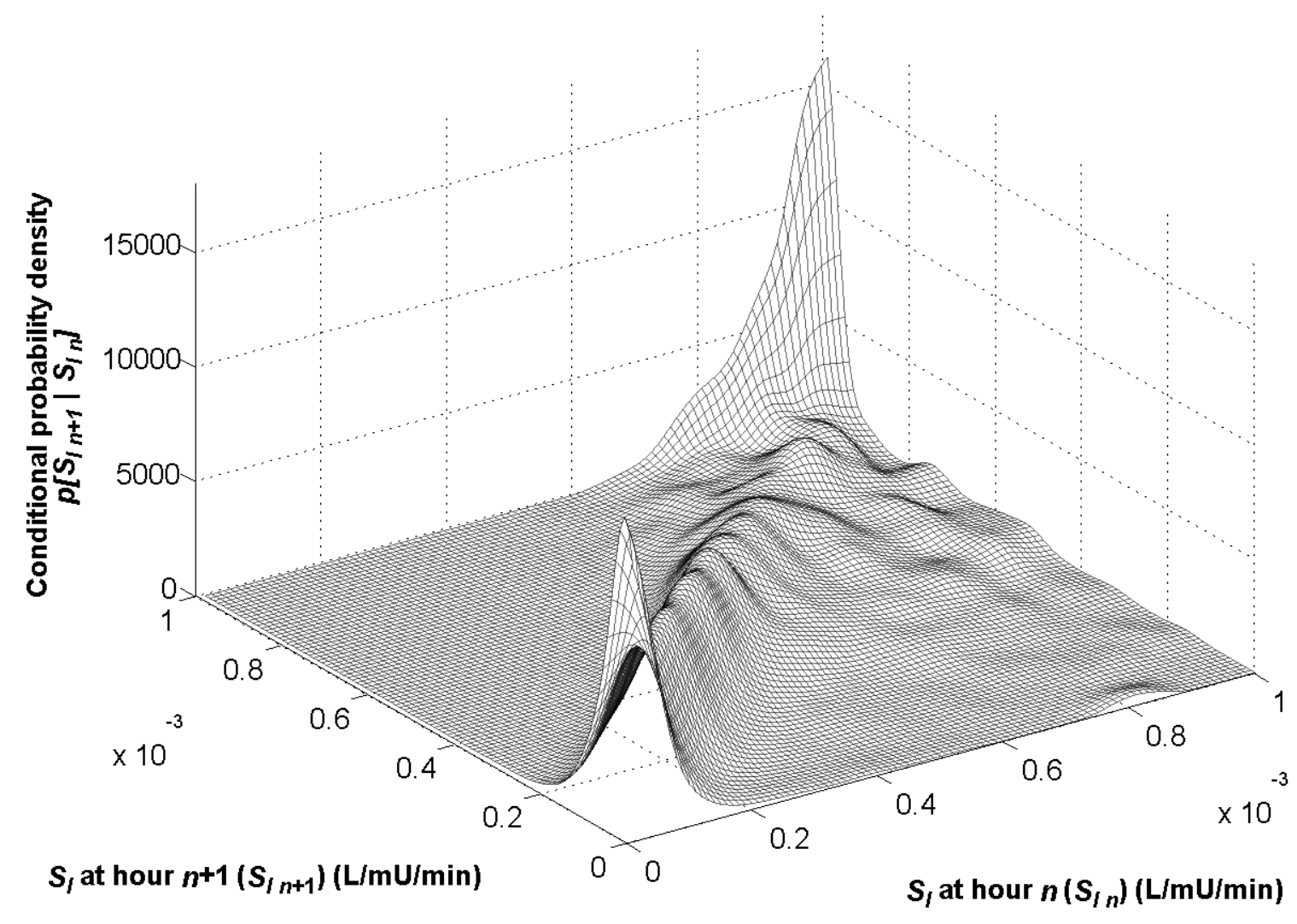

Figure 2. Stochastic model of $S_{I}$ variability. Conditional probability density functions are the surface slices along $S_{I n+l}$ axis, each slice has an area under the curve summing to 1.0 . 


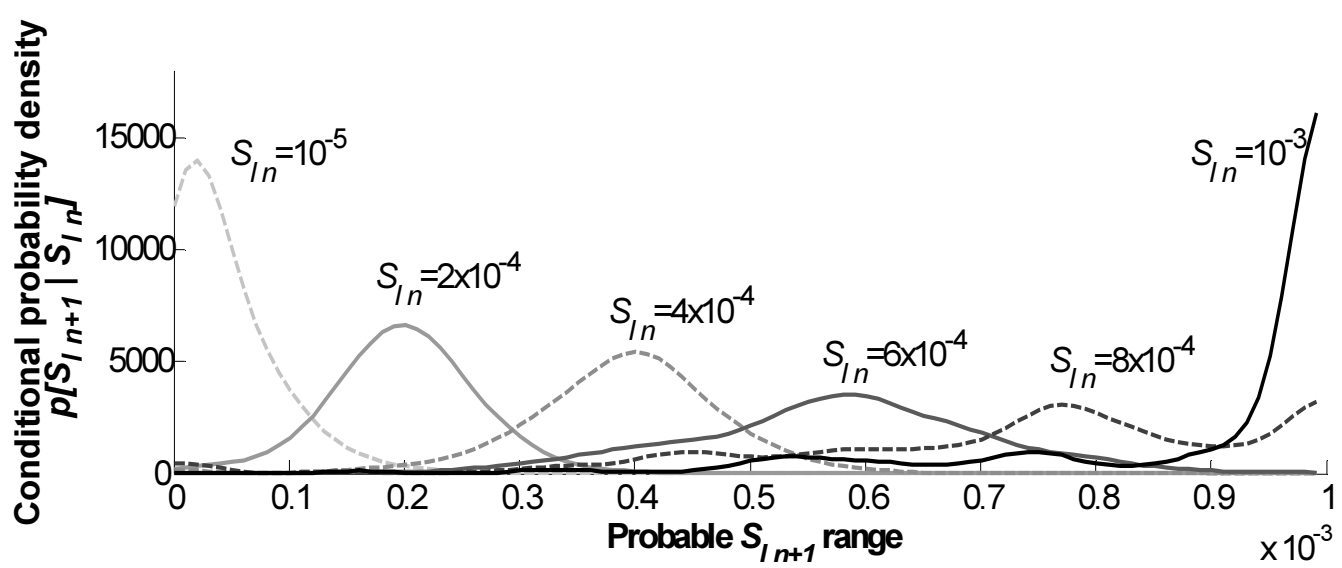

Figure 3. Cascade of $S_{I}$ probability density function slices over the fitted $S_{I}$ range. 


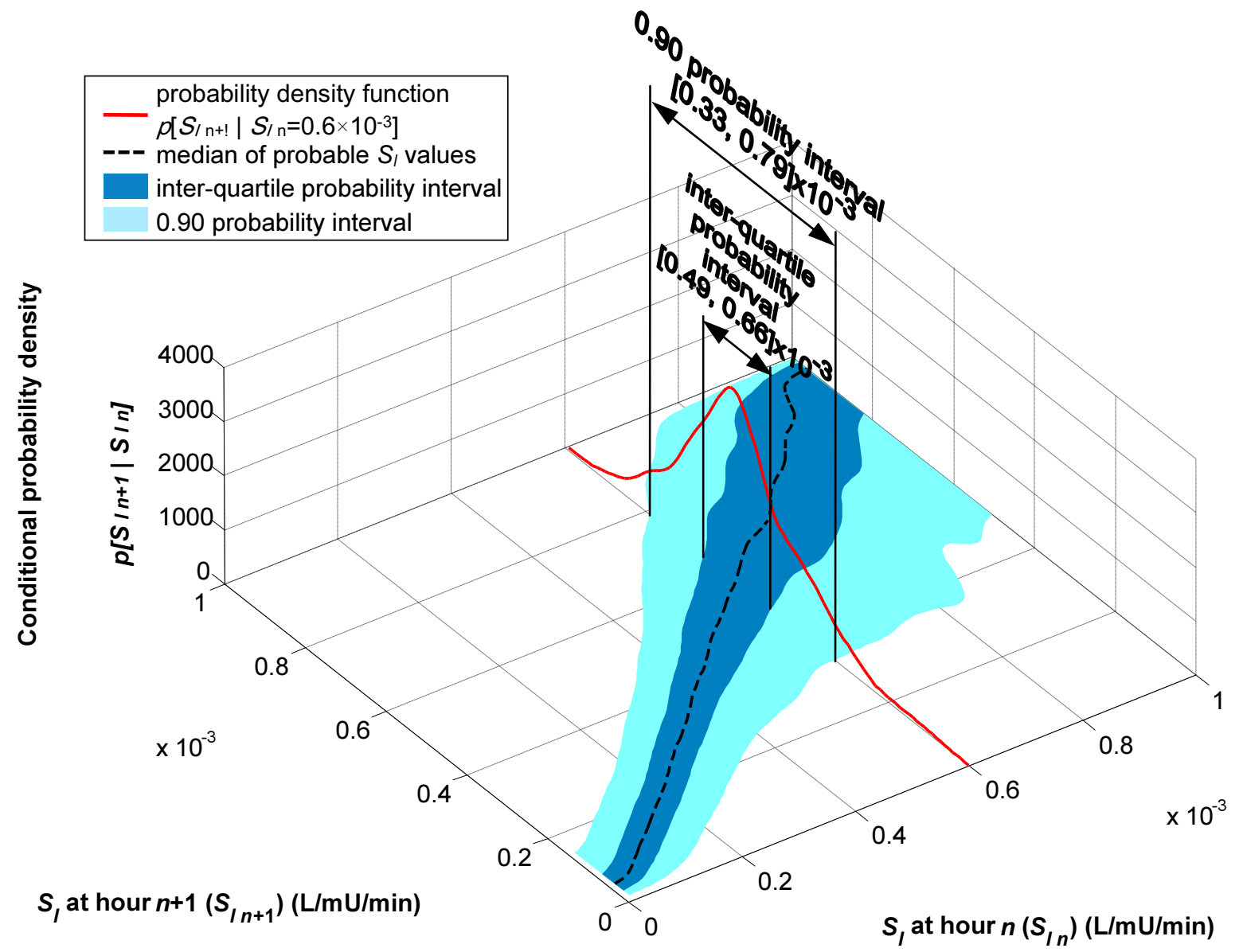

Figure 4. Probability density function of potential $S_{I}$. Probability intervals across the model space from Figure 2 are shown by shaded areas. 

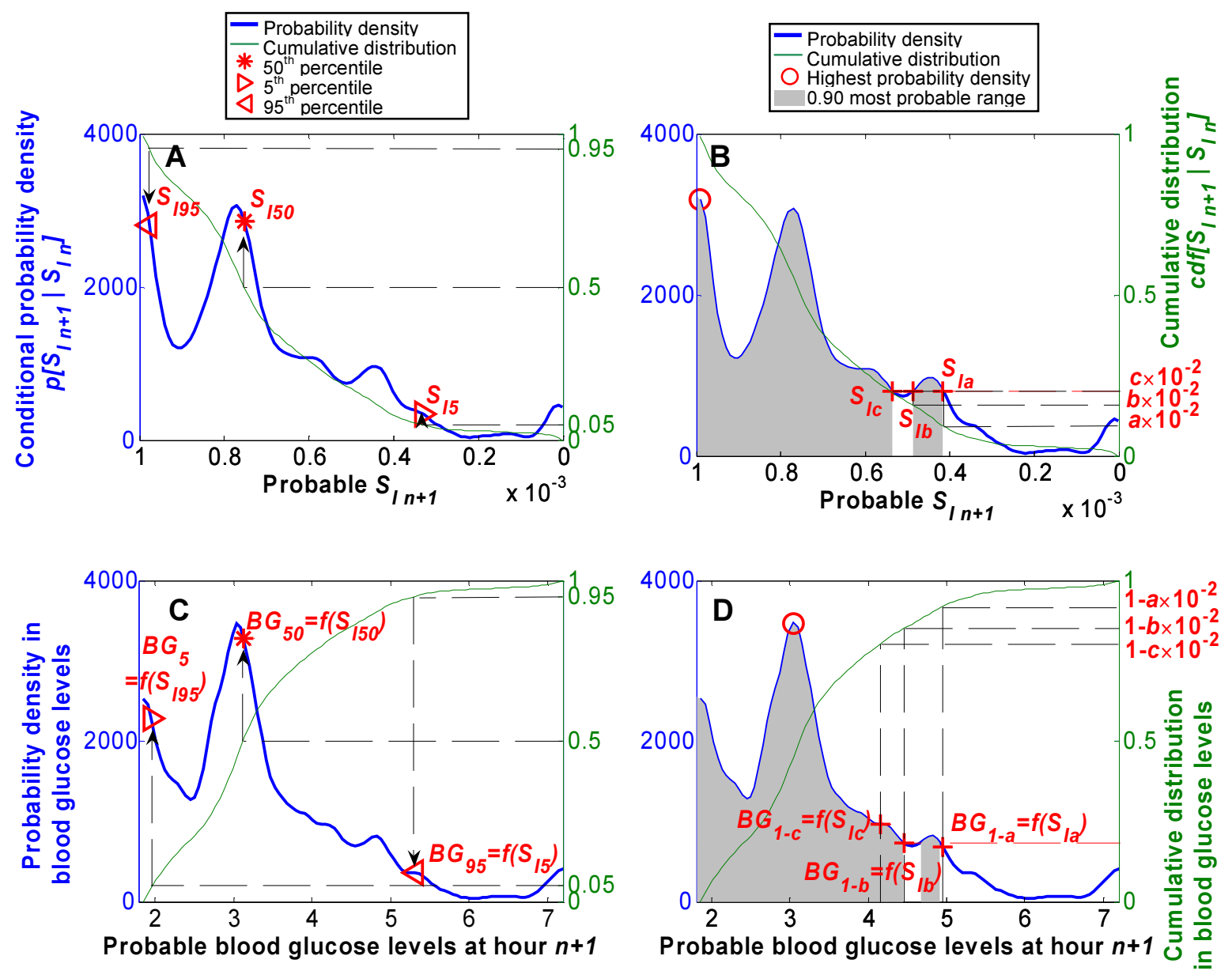

Figure 5. Probability density transition from $S_{I}$ to blood glucose levels. Panels A and B show the pdf (right axis) and the cdf (left axis) in $S_{I n+l}$, when $S_{I n}=8 \times 10^{-4}$ is known. Panels C and D show the resulting pdf (right axis) and cdf (left axis) in blood glucose levels at hour $n+1\left(B G_{n+1}[\mathrm{mmol} / \mathrm{L}]\right)$ through Monte Carlo Simulation. The transformation through Equations (1)-(5) from upper panels to lower panels is denoted by $f$. The shaded areas in panel $\mathrm{B}$ and $\mathrm{D}$ have the highest $90 \%$ probability. The $90 \%$ most probable $S_{I n+l}$ intervals in panel B are between cdf $=\left[\mathrm{a} \times 10^{-2}, \mathrm{~b} \times 10^{-2}\right]$ and $\left[\mathrm{c} \times 10^{-2}, 1.0\right]$. Note that higher $S_{I}$ values in the left hand side of the reversed x-axis in panels $\mathrm{A}$ and $\mathrm{B}$ results in higher $B G$ values on the right hand side in panels $\mathrm{C}$ and D. 

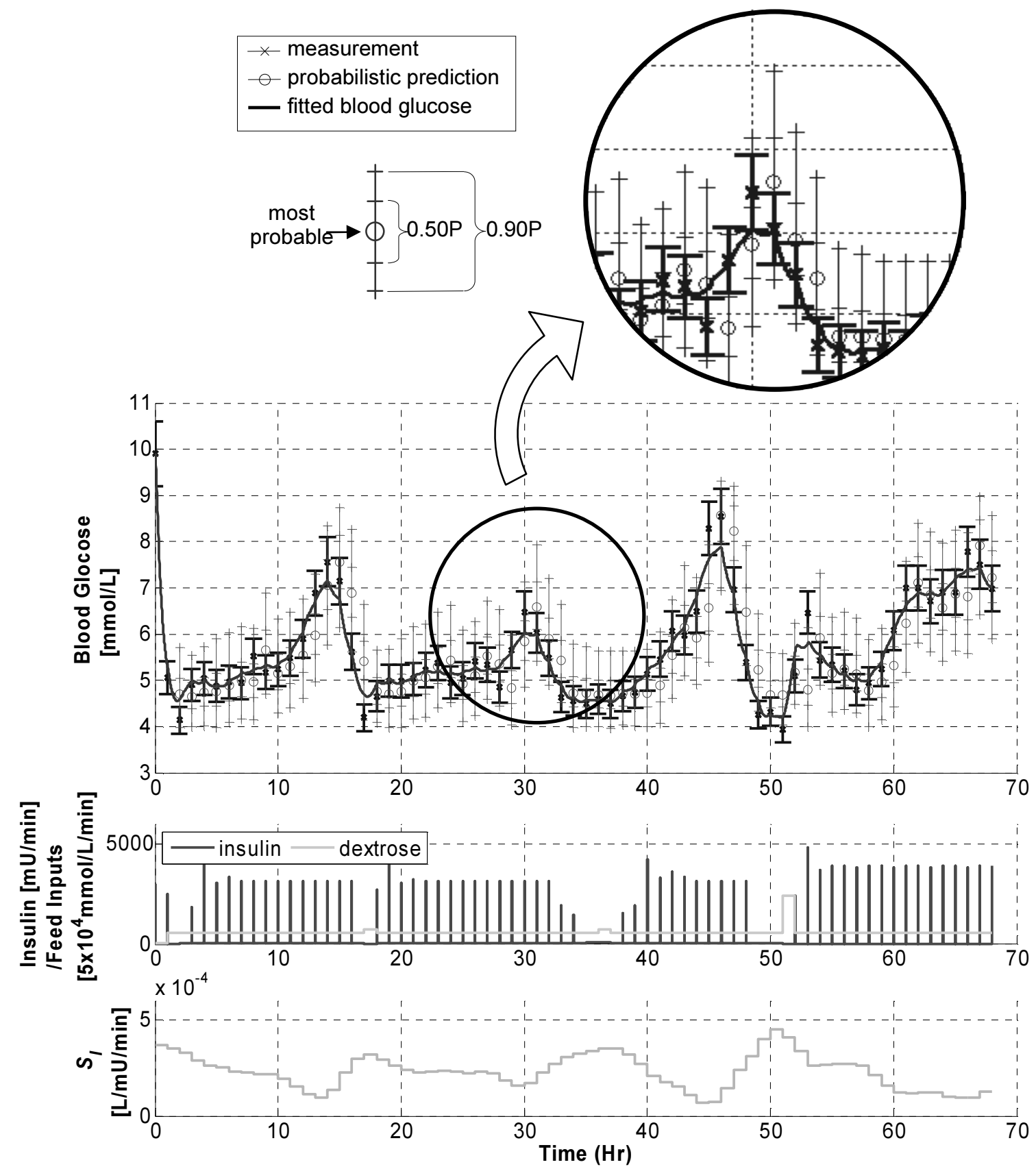

Figure 6. Example of a virtual trial. The top panel displays the blood glucose evolution, where bold crosses are virtually generated blood glucose levels are one hour intervals, with thick bars indicating measurement error. The controller predicted blood glucose levels are shown in circles, with thin bars indicating their probability intervals. 

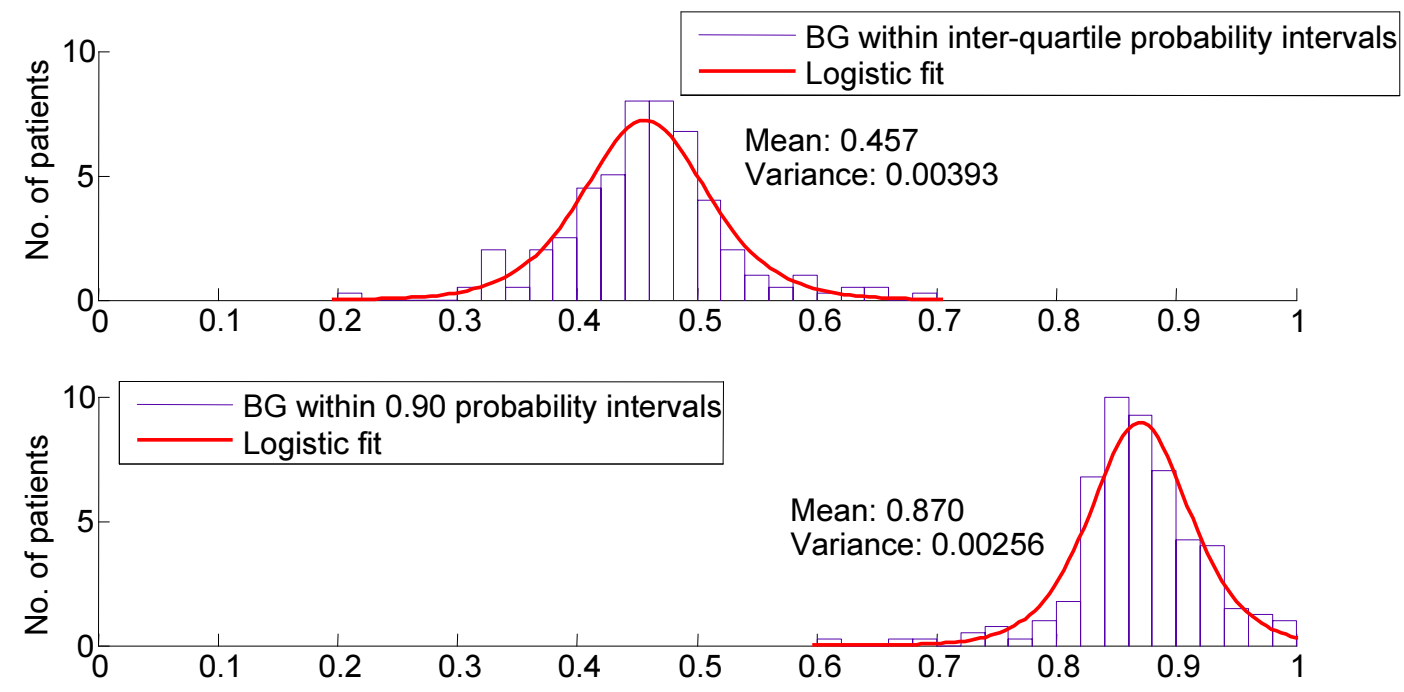

Figure 7. Probability interval analysis per patient. Plotted are the individual patient averages. The upper plot is the fraction of measurements in the inter-quartile interval. The bottom panel is the fraction in the 0.90 probability interval. 


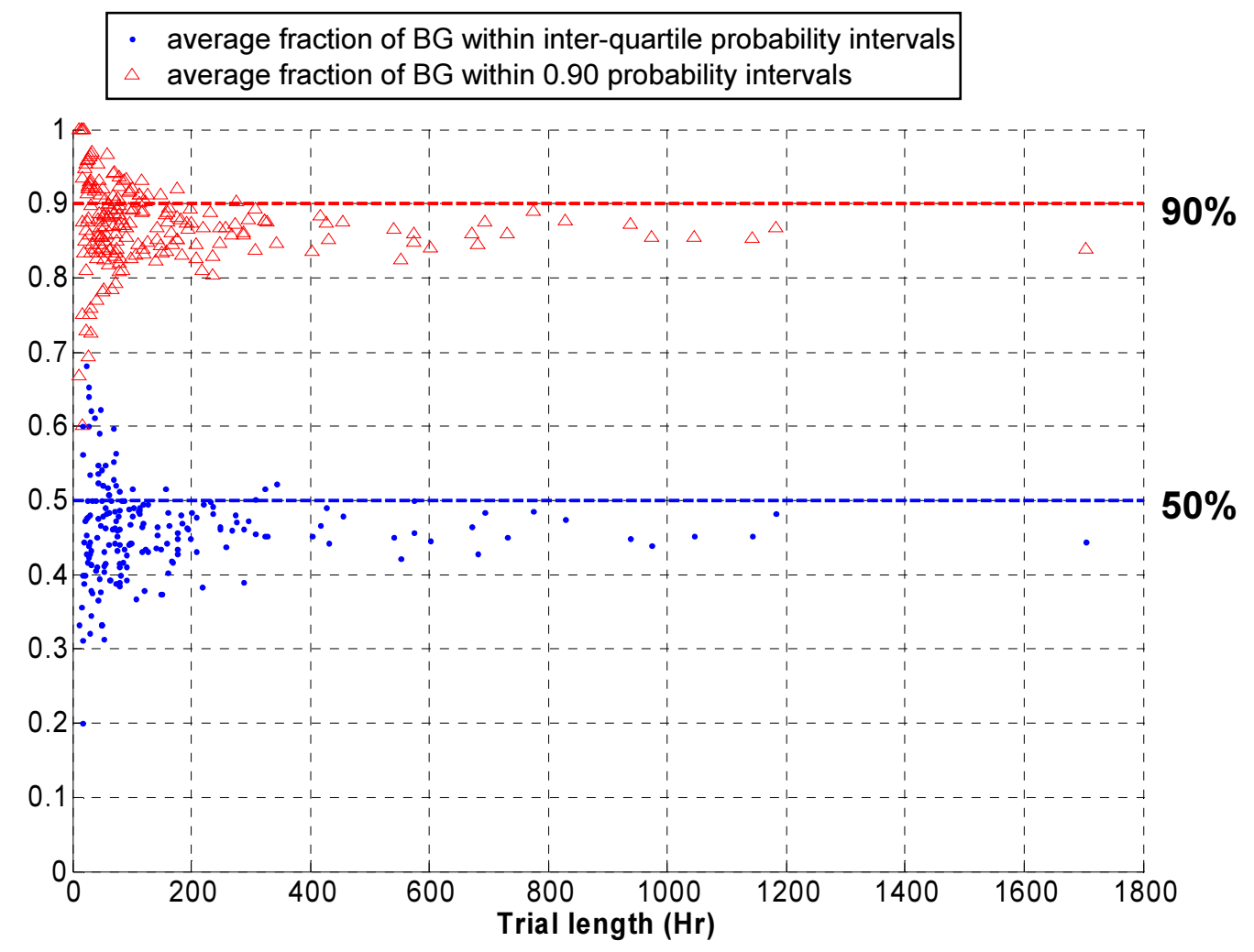

Figure 8. Virtual trial length versus probability interval accuracy. 


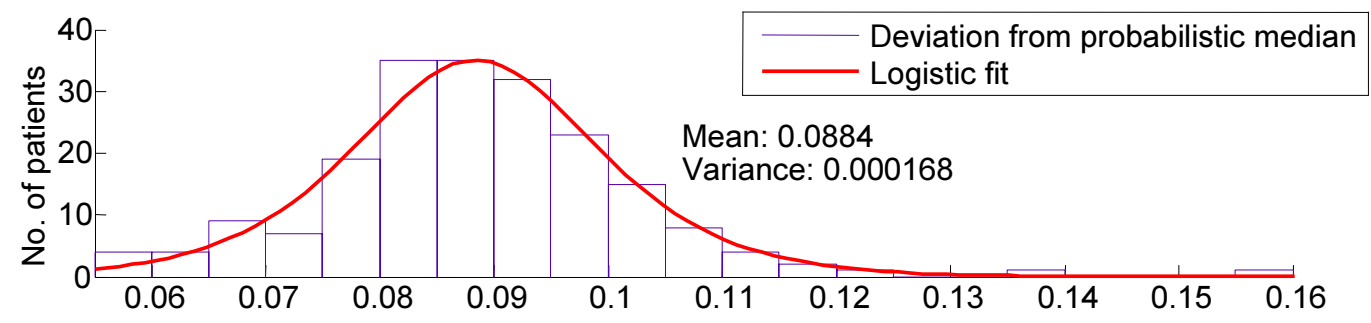

Figure 9. Percentage deviation of virtual trial blood glucose levels from probabilistic medians. 


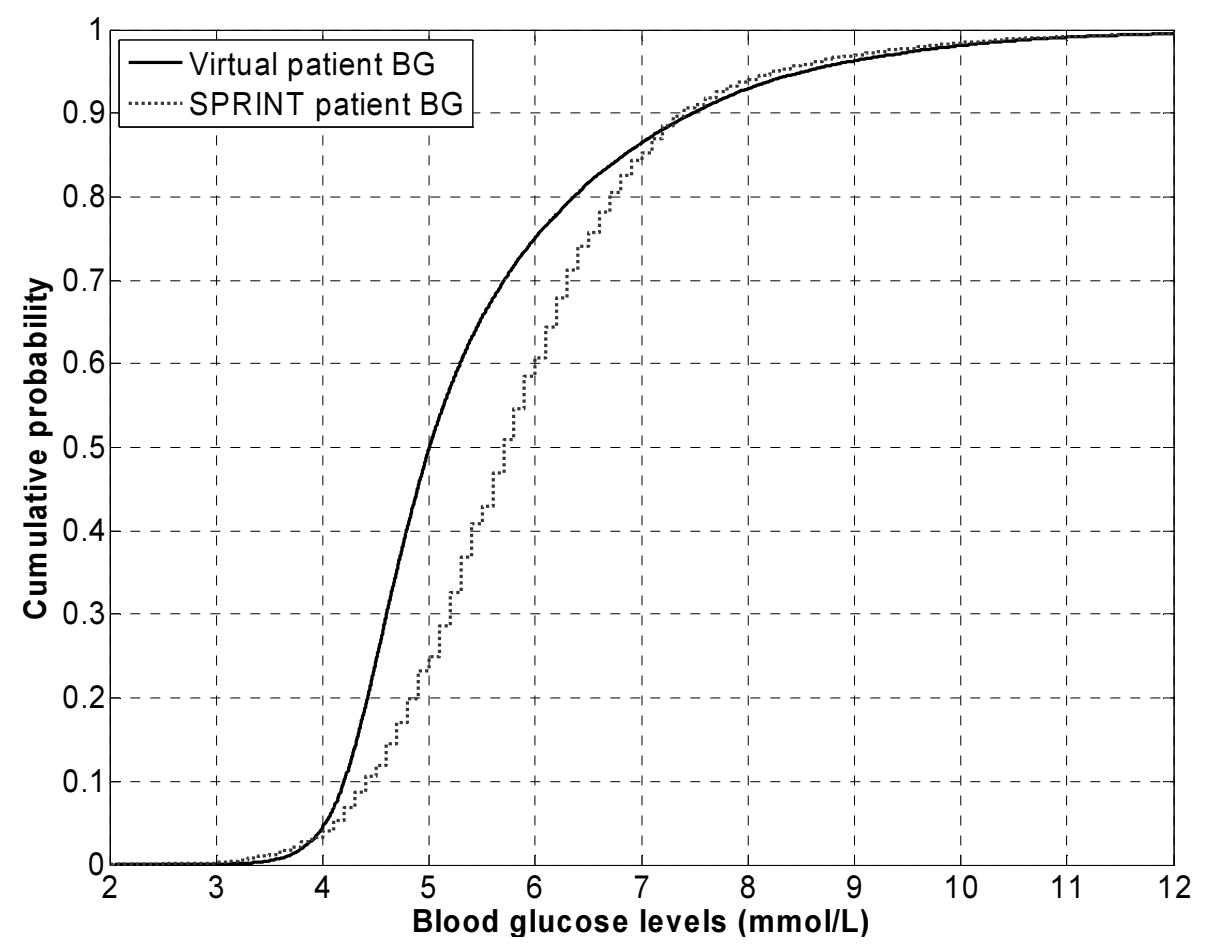

Figure 10. Blood glucose measurement distribution comparison between clinical SPRINT results and virtual patients under the improved stochastic controller presented. 
University of Canterbury

Private Bag 4800

Christchurch
Telephone: +64-3-366 7001

Facsimile: $\quad+64-3-3642078$

Website: www.mech.canterbury.ac.nz

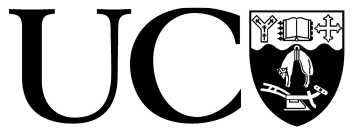

UNIVERSITY OF CANTERBURY

\section{Dear Prof Carson,}

Re-submitted is our manuscript entitled: "Stochastic Modelling of Insulin Sensitivity and Adaptive Glycemic Control for Critical Care". The paper presents the application of Markov modelling to create a stochastic model of insulin sensitivity variability in critical care glycemic control. The model can then be used clinically, as shown, to help optimise decision making during the provision of model-based intensive insulin therapy.

The re-submitted manuscript has been modified to address the two reviewers' comments. A general comment between both reviewers is on the stochastic model probability intervals. As both reviewers pointed out, the probability density functions are asymmetric near stochastic model boundaries. Therefore, it is important to discuss the implication and the impact of having these asymmetries in the model space. The discussion on probability intervals, their calculation, and clinical feasibility are extensively revised in Section 3.2. The manuscript is now better structured, and should be easier to follow. Finally, the manuscript offers a solution to the computational intensity of exact probability intervals, which is the use of equal-tailed probability interval estimates for clinical decision support. This solution provides fast, and therefore clinically useful glycemic control assistance, and is adequately accurate with minimal losses of $1-4 \%$ in exactness.

Specific reply to each reviewers' comment is attached with this submission. We thank the reviewers for their input and efforts. The manuscript should now be more solid, with issues raised more clearly addressed. In particular, the link between text and figures is now stronger, with figure captions expanded to be more self-explanatory.

The re-submitted manuscript, however has grown in length, to strengthen and address each reviewer's commented issues. The manuscript now consists of 24 pages of text, 4 pages of references, with 5 tables, and 10 figures in the end. Each page is double spaced and has around 250 words equivalent per page. The total equivalent length is now approximately 9,000 words. All work submitted in this manuscript is original.

Again, thank you for your time and consideration of our manuscript. We look forward to your response.

Regards,

Jessica Lin

Research Assistant

Centre for Bio-Engineering/Dept of Mechanical Engineering

University of Canterbury

Christchurch, New Zealand

ph: +64 33642987 ext 7486 fax: +64 33642078 


\section{Reply to reviewer \#1}

\section{General comments:}

The reviewer commented that the problems at the tails of the insulin sensitivity probability distribution "could be probably avoided by allowing, for instance, endogenous glucose production to present some stochastic variability." The authors thank the reviewer for pointing this out. Although many detailed discussions on the physiological model were omitted in this manuscript due to length restriction, this point should be made clearer to justify the methodology. The authors have modified the last paragraph of Section 3.1, and also added another 2 paragraphs after it, to clarify and address this point:

"Across the fitted range of $S_{I}$, the conditional probability density function is largely uni-modal and symmetric. However, as shown in the cascade plot of conditional $S_{I n+1}$ probability density functions across the $S_{I n}$ model range in Figure 3, there are some numerical artefacts near the boundaries of the fitted range. Probability density functions for $S_{I n}$ peak at boundary values because of the fitting constraints. For $S_{I n}=$ $\left[1 \times 10^{-5}, 1 \times 10^{-4}\right]$ and $\left[0.75 \times 10^{-3}, 1 \times 10^{-3}\right]$ at the edges of Figure 3 , the percentile values away from the median do not necessarily decrease in probability. These ranges span $34 \%$ of the fitting range $\left[1 \times 10^{-5}, 1 \times 10^{-3}\right]$, in which $20 \%$ of the SPRINT data falls (18\% between $S_{I n}=\left[1 \times 10^{-5}, 1 \times 10^{-4}\right]$ and $2 \%$ between $\left[0.75 \times 10^{-3}, 1 \times 10^{-3}\right]$ in Figure 1). Note that Figures 2 and 3 show the conditional probability density functions which are scaled to have the area under each function summing to 1 . Thus, the asymmetry becomes very pronounced in Figures 2 and 3. The probability of $S_{I}$ taking on these asymmetric conditional probability density functions is in fact very low in the overall joint probability density function shown in Figure 1, where the probability density sums to 1 over the entire fitting surface.

The asymmetric $S_{I}$ probability density functions could perhaps be due to other not explicitly modelled physiology, such as variations in endogenous glucose production. More specifically, "bumpy" areas of $S_{I}$ probability density function in Figure 3 may be a sign of $S_{I}$ absorbing physiology unmodelled or undermodelled in Equations (1)(5). However, this unmodelled physiology represents uncommon sudden, or extreme, dynamics, evident by the low overall joint probability density in these areas in Figure 1. Practically, to include all such variations in the glucose-insulin model would make it overly complicated and it would thus lose clinical feasibility. Such additions would also require added assumptions for endogenous insulin or glucose production that are not clinically measured in real-time.

Assumptions for these responses could also compromise control accuracy, as both endogenous insulin and glucose productions are known to vary significantly between patients [25-29] and over time. In addition, endogenous glucose production is suppressed with significant insulin administration in both normal and stressed states [30], which is the case for critically ill patients under insulin therapy for glycemic management. Similarly, the endogenous insulin production is effectively removed or significantly reduced in the presence of significant exogenous insulin [29, 31-33], not to mention its inhibition in stress hyperglycemia [e.g. 34]. It is therefore, in the interest of this model-based clinical control applications, to have these effects mitigated into $S_{I}$. In $[10,12,15]$, the model of Equations (1)-(5) and the impact of mitigated effects are discussed in detail. The result is a much more clinically feasible 
control model, where $S_{I}$ accounts for critically ill population's overall sensitivity to insulin and/or its utilisation."

Specific comments:

1. The following two sentences regarding stress-induced hyperglycemia are added after the first sentence in the first paragraph of Section 1.

"The increased counter-regulatory hormone and cytokine response stimulates endogenous glucose production and increases effective insulin resistance. Absolute and relative insulin deficiency and steroid-based therapies are further causes."

The reviewer suggested discussion on the possible implications of ignoring endogenous glucose production is added to Section 3.1, as per the reply to the general comments.

2. The introduction sentence now reads:

"A clinically verified patient-specific glucose-insulin metabolic model [10, 12, 14 , $15]$ is used to account for time-varying insulin sensitivity in critical care."

3. Paragraphs after Equations (1)-(5) in Section 2.1 now read:

"where $G[\mathrm{mmol} / \mathrm{L}]$ denotes glucose above an equilibrium level $G_{E}[\mathrm{mmol} / \mathrm{L}]$, and $I[m U / L]$ denotes the plasma insulin from an exogenous insulin input. Insulin utilization over time is captured by $Q[\mathrm{mU} / \mathrm{L}]$, where $k[1 / \mathrm{min}]$ is the effective insulin half-life parameter. Patient endogenous glucose removal and insulin sensitivity are $p_{G}[1 / \mathrm{min}]$ and $S_{I}[\mathrm{~L} / \mathrm{mU} / \mathrm{min}]$ respectively. The parameter $V_{I}[L]$ is the insulin distribution volume and $n[1 / \mathrm{min}]$ is the first order decay rate for plasma insulin. External nutrition and insulin inputs are $P(t)[\mathrm{mmol} / \mathrm{L} / \mathrm{min}]$ and $u_{e x}(t)[\mathrm{mU} / \mathrm{min}]$, respectively. Michaelis-Menten parameters $\alpha_{I}[L / m U]$ and $\alpha_{G}$ $[L / m U]$ define plasma insulin disappearance saturation and insulin-stimulated glucose removal saturation, respectively. The exogenous glucose appearance and decay rate used to define $P(t)$ are $k_{p r}$ and $k_{p d}$. Specific details on physiological model dynamics are evaluated in $[10,12,16]$.

In Equation (1), the saturation mechanism on insulin effect creates a unique index of insulin sensitivity, $S_{I}$, compared to other model-based measures. The result is an $S_{I}$ index that more closely approximates the effective net tissue sensitivity to insulin, and its variation to the evolution of patient condition and drug therapy. It has also been shown to drive patient response to intervention and thus control efficacy in prior studies $[10,12]$. This model measure is also highly correlated to clamp-derived ISI over 146 patients [17-19]. Hence, understanding variation in $S_{I}$ should enable more effective control."

Due to the length restriction for the manuscript, detailed aspects of the physiological model is referred to previous publications.

4. The following paragraphs are also added to the end of Section 3.1 to discuss the

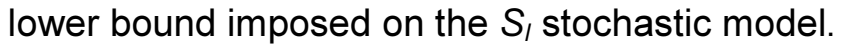


"In Figure 1, the lower bound for $S_{I}$ has greater influence on the overall stochastic model than the upper bound. However, no patients have fitted $S_{I}$ staying at the lower bound for a prolonged period, again suggesting that these occasions are more sudden, short term condition, such as suppressed insulin sensitivity or utilisation due to drug therapies, rather than long term, or gradual changes, such as increased glucose production. During numerical fitting, if fitted $S_{I}$ stays at the lower bound for a prolonged period of 5 hours, the fitting method recognises a gradual evolution, and adjusts $G_{E}$ to address factors such as endogenous glucose production. During the total of 23,324 control hours, only 39 such occurrences were observed, which is $0.17 \%$ of the time.

The short term changes, although causes fitted $S_{I}$ to hit the lower bound, if unconstraint, will often result in negative $S_{I}$ because of significant drug effects or acute medical conditions such as atrial fibrillation [12]. In addition, increased endogenous production with inhibited glucose production can result in an effectively zero or negative modelled $S_{I}$. These cases are too wide ranged to be accounted for in the physiological model. Note that an evolving $G_{E}$ that is too high leads to a reduction in modelled endogenous glucose production, and a higher $S_{I}$ may also result in the model due to a lower $-p_{G} G$ term. Mitigating the impact of these events into fitted $S_{I}$ simplifies the model to be clinically control feasible.

In addition, incorrect modelling of these conditions which are difficult to account for can severely compromise patient safety. More specifically, underestimating $S_{I}$ can lead to excess insulin being given. With the aim of applying the physiological and stochastic models in clinical control, the lower bound of $S_{I}$ has an important role. Finally, the fitted $S_{I}$, with higher data concentration around the lower bound, realistically reflects the highly variable dynamics in the critical care environment, where drug therapies and acute medical conditions that result in highly resistant patients are common."

5. Section 3.2 discusses and explains the, in fact, simple applicability of the stochastic model that, at first glance, may have appeared too complex to be clinically useful given its asymmetric distributions near the model boundaries. This section employs Figure 5 to illustrate the implication of using the easy-tocalculate equal-tailed probability intervals. The equal-tailed probability intervals although do not provide the exact solutions to the most probable intervals, they do provide satisfactory solutions at an incomparably advantageous speed over Monte Carlo simulation method, and therefore makes the stochastic model clinically feasible for control applications.

Section 3.2 has been re-written and broken into two subsections to make it easier to follow. The probability intervals are now identified in Figure 4. The differences between equal-tailed and exact probability intervals are further explained, with a clearer tie to Figure 5. A new table, now Table 2, is added to make the comparison even more straight forward.

Regarding to the use of equal-tailed probability intervals in clinical decision making, while asymmetry exists near model boundaries, for the majority of the model pace, the probability density functions are near-symmetric. Therefore, equal-tailed probability intervals do, a large majority of the time, provide the most probable forecast for the clinical outcome, with small errors being accepted in other instances for the sake of computational simplicity. In addition, equal-tailed 
probability intervals are generally well accepted and most familiar to clinicians over other forms of expressions.

6. APACHE = Acute Physiology and Chronic Health Evaluation. This is now added to the text.

7. Step 6 is modified to read:

"Return to step 1 with $S_{I n+l}$ being the new $S_{I n \text {." }}$

And a paragraph is added after step 6 to clarify virtual $S$, generation:

"Essentially, a virtual patient's $S_{I}$ evolution follows the joint probability density contour in Figure 1, making its way to the highest probability density regions. Each $S_{I n+l}$ is dependent on the previous state, $S_{I n}$, where the probability density function of of $S_{I n+l}$ is defined in Equations (10)-(11). Hence, in step 1 above, $S_{I}$ takes a walk to a point, say $y$, along the probability density function curve $p\left(S_{I n+I}=y \mid S_{I n}=x\right)$ as shown in Figure 4. Step 6 then "walk" the $S_{I}$ across (along $S_{I n+l}$ axis) to where $S_{I n}=y$, and the process repeats."

8. Section 3.4 is now broken into 3.4.1 and 3.4.2.

9. Thickness of bars are now noted in the text. More explanation is also added to the figure caption.

10. Virtual trial lengths are generated according to data from SPRINT patient cohort, as explained in Section 3.4.1, "Trial lengths were also randomly generated to create a similar statistical distribution to that in the SPRINT trials."

To clarify the relationship between trial length and control accuracy, the $4^{\text {th }}$ line now reads:

"Figure 8 illustrates how shorter trials can produce outliers, where most variability is gone after 50-75 hours, indicating stabilised patient condition under long term, tight glycemic control with little variability in glycemic level."

11. This line now reads:

"The percentage lower than $4 \mathrm{mmol} / \mathrm{L}$ is slightly increased from $3.3 \%$ to $4.5 \%$, with $0 \%$ under $2.5 \mathrm{mmol} / \mathrm{L} . "$

12. The $2^{\text {nd }}$ part of the legend is now in the footnote of Table 5 (which used to be Table 4).

13. The authors feel that the use of these figures is important for explaining different issues raised in the manuscript. In addition, contours are now added in Figure 1, and used to explain virtual patient generation. Figure 2 provides a whole picture of the stochastic model. Figure 3 is used to bring out the shapes of probability density function across the model space, and therefore acts as an important lead into the discussion on probability intervals. Figure 4 demonstrates the derivation of probability intervals from probability density functions, and shows the probability intervals across the model space. 
Therefore, the authors do not wish to omit any of these figures. However, more information is added to the figure captions and/or legend to make their difference in form and function more self-explanatory.

The authors can condense as required but feel there will be a loss of clarity. Therefore, the authors leave this decision to the editor's discretion.

14. Better linkage between text and the Figure is now made, and more information is added to the figure caption.

15. Figure 10 now compares the cumulative distribution of virtual trial and SPRINT trial blood glucose levels, and the horizontal axis now has a more suitable limits.

16. Additional information has been added to figures to make them more selfexplanatory.

Reviewer \#2:

\section{General Comments:}

Reviewer \#2's general comment regards Section 3.2, where the probability interval calculations and clinical use are discussed. The exact method for calculating probability intervals was presented. A clinically feasible solution to the exact method was then offered that produce little errors with a fast clinical delivery time. This section employs Figure 5 to illustrate the implication of using the easy-to-calculate equal-tailed probability intervals. The equal-tailed probability intervals although do not provide the exact solutions to the most probable intervals, they do provide satisfactory solutions at an incomparably advantageous speed over Monte Carlo simulation method, and therefore makes the stochastic model clinically feasible for control applications.

Section 3.2 has been re-written and broken into two subsections to make it easier to follow. The probability intervals are now identified in Figure 4. The differences between equal-tailed and exact probability intervals are further explained, with a clearer tie to Figure 5. Better links between Figure 5 and text are also made, with more information added to the figure caption. Finally, a new table, now Table 2, is added to make the comparison even more straight forward.

Specific comments:

(i) The symbol " $\mathrm{P}$ " is now changed to lower case " $\mathrm{p}$ " to stand for probability densities.

(ii) As shown in Figure 3, the maximum probability density for each conditional probability density functions across $S_{\mid n+1}$ varies. In particular, the curve in Figure 4 is the $S_{I n}=6 \times 10^{-4}$ curve shown in Figure 3 , and the curve in the upper panels in Figure 5 is the $S_{I n}=8 \times 10^{-4}$ curve shown in Figure 3. Therefore, Figures 4 and 5 have lower limits for the vertical axes.

(iii) The spelling is now corrected.

(iv) As per the text in now Section 3.2.1, $4^{\text {th }}$ paragraph, last sentence, 
"Note that in panels A and B, the X-axes are decreasing from left to right, producing a similar shaped probability density function in blood glucose levels in panels C and D with blood glucose levels increasing as $S_{I n+l}$ decreases",

the right-pointing triangles show the $5^{\text {th }}$ percentiles, and the left-pointing triangles show the $95^{\text {th }}$ percentiles in both distributions. This is also noted in the Figure legend.

(v) These unshaded regions have lower probability density than shaded regions, as pointed out in the text in Section 3.2.1,

“..., the $90 \%$ most probable $S_{I n+I}$ values are identified by the shaded areas in panel B. These values are discontinuous, and have higher probability of occurring than values outside the shaded areas",

and in the bullet points following this passage.

(vi) The reviewer has mistaken the multiplication symbol as variable " $x$ ". A different font is chosen to make the multiplication symbol more obviously identified.

\section{Other Comments:}

1. the sentence now reads:

"Over 165 patients and 23,000 patient hours, it achieved an average blood glucose level of $5.8 \pm 1.2 \mathrm{mmol} / \mathrm{L}$ with $61 \%$ of the time in the $4-6.1 \mathrm{mmol} / \mathrm{L}$ band [4]"

where "mmol/L" is a commonly accepted unit for measuring blood glucose levels.

2. $k_{p r}$ and $k_{p d}$ are The exogenous glucose appearance and decay rate used to define the glucose appearance $P(t)$. This is now added in the paragraph after Equations (1)-(5) in Section 2.1.

3. This has been modified to the reviewer's suggestion.

4. This figure is a cascade plot of the probability density functions across the model space. This figure shows how shape of the probability density functions changes across the fitting space. This is explained in the $3^{\text {rd }}$ to last paragraph in Section 3.1 (used to be the last paragraph). The model space has lower limit of $10^{-5}$ and $10^{-3}$, thus Figure 3 has the same limits for its horizontal axis.

5. The reference style has been corrected.

6. Contour curves are now added to the figure to aid readability. The authors suspect that the "purple" referred to by the reviewer are actually dots of fitted data. These dots are now made bigger to ensure readability in print.

The scale of the colour map and the contours has a maximum of $5.5 \times 10^{11}$ because the joint probability density function sums to 1 over the entire fitting surface. This is explained in the $3^{\text {rd }}$ to last paragraph in Section 3.1 (used to be the last paragraph). Because the values for $S_{\text {I }}$ are small $\left(10^{-5}\right.$ to $\left.10^{-3}\right)$, the "height" of the joint probability density function in the model space is thus great.

7. This has been corrected. 